\title{
Algorithms of common solutions for a variational inequality, a split equilibrium problem and a hierarchical fixed point problem
}

\section{Abdellah Bnouhachem*}

Dedicated to Professor Bingsheng He on the occasion of his sixty-fifth birthday

${ }^{*}$ Correspondence:

babedallah@yahoo.com

School of Management Science and

Engineering, Nanjing University,

Nanjing, 210093, P.R. China

ENSA, Ibn Zohr University, Agadir,

BP 1136, Morocco

\begin{abstract}
In this paper, we suggest and analyze an iterative scheme for finding an approximate element of the common set of solutions of a split equilibrium problem, a variational inequality problem and a hierarchical fixed point problem in a real Hilbert space. We also consider the strong convergence of the proposed method under some conditions. Results proved in this paper may be viewed as an improvement and refinement of the previously known results.

MSC: 49J30; 47H09; 47J20
\end{abstract}

Keywords: split equilibrium problem; variational inequality problem; hierarchical fixed point problem; projection method; strictly pseudo-contractive mapping

\section{Introduction}

Let $H$ be a real Hilbert space, whose inner product and norm are denoted by $\langle\cdot, \cdot\rangle$ and $\|\cdot\|$. Let $C$ be a nonempty closed convex subset of $H$ and $D$ be a mapping from $C$ into $H$. A classical variational inequality problem, denoted by $V I(D, C)$, is to find a vector $u \in C$ such that

$$
\langle v-u, D u\rangle \geq 0, \quad \forall v \in C .
$$

The solution of $\operatorname{VI}(D, C)$ is denoted by $\Omega^{*}$. It is easy to observe that

$$
u^{*} \in \Omega^{*} \quad \Longleftrightarrow \quad u^{*}=P_{C}\left[u^{*}-\lambda D u^{*}\right], \quad \text { where } \lambda>0 \text {. }
$$

This alternative formulation has played a significant part in developing various projectiontype methods for solving variational inequalities. We now have a variety of techniques to suggest and analyze various iterative algorithms for solving variational inequalities and the related optimization problems; see [1-29].

We introduce the following definitions which are useful in the following analysis.

Definition 1.1 The mapping $T: C \rightarrow H$ is said to be

\section{Springer}

(C2013 Bnouhachem; licensee Springer. This is an Open Access article distributed under the terms of the Creative Commons Attribution License (http://creativecommons.org/licenses/by/2.0), which permits unrestricted use, distribution, and reproduction in any medium, provided the original work is properly cited. 
(a) monotone if

$$
\langle T x-T y, x-y\rangle \geq 0, \quad \forall x, y \in C
$$

(b) strongly monotone if there exists $\alpha>0$ such that

$$
\langle T x-T y, x-y\rangle \geq \alpha\|x-y\|^{2}, \quad \forall x, y \in C ;
$$

(c) $\alpha$-inverse strongly monotone if there exists $\alpha>0$ such that

$$
\langle T x-T y, x-y\rangle \geq \alpha\|T x-T y\|^{2}, \quad \forall x, y \in C ;
$$

(d) nonexpansive if

$$
\|T x-T y\| \leq\|x-y\|, \quad \forall x, y \in C ;
$$

(e) $k$-Lipschitz continuous if there exists a constant $k>0$ such that

$$
\|T x-T y\| \leq k\|x-y\|, \quad \forall x, y \in C
$$

(f) contraction on $C$ if there exists a constant $0 \leq k<1$ such that

$$
\|T x-T y\| \leq k\|x-y\|, \quad \forall x, y \in C .
$$

It is easy to observe that every $\alpha$-inverse strongly monotone $T$ is monotone and Lipschitz continuous. It is well known that every nonexpansive operator $T: H_{1} \rightarrow H_{1}$ satisfies, for all $(x, y) \in H_{1} \times H_{1}$, the inequality

$$
\langle(x-T(x))-(y-T(y)), T(y)-T(x)\rangle \leq \frac{1}{2}\|(T(x)-x)-(T(y)-y)\|^{2}
$$

and therefore we get, for all $(x, y) \in H_{1} \times F(T)$,

$$
\langle x-T(x), y-T(x)\rangle \leq \frac{1}{2}\|T(x)-x\|^{2}
$$

see, e.g., [9], Theorem 1 and [10], Theorem 3.

A mapping $T: C \rightarrow H$ is called a $k$-strict pseudo-contraction if there exists a constant $0 \leq k<1$ such that

$$
\|T x-T y\|^{2} \leq\|x-y\|^{2}+k\|(I-T) x-(I-T) y\|^{2}, \quad \forall x, y \in C .
$$

The fixed point problem for the mapping $T$ is to find $x \in C$ such that

$$
T x=x
$$

We denote by $F(T)$ the set of solutions of (5). It is well known that the class of strict pseudocontractions strictly includes the class of nonexpansive mappings, then $F(T)$ is closed and convex and $P_{F(T)}$ is well defined (see [29]). 
The equilibrium problem denoted by $E P$ is to find $x \in C$ such that

$$
F(x, y) \geq 0, \quad \forall y \in C
$$

The solution set of (6) is denoted by $E P(F)$. Numerous problems in physics, optimization and economics reduce to finding a solution of (6); see [7, 12, 23, 24]. In 1997, Combettes and Hirstoaga [8] introduced an iterative scheme of finding the best approximation to the initial data when $E P(F)$ is nonempty. Recently Plubtieng and Punpaeng [23] introduced an iterative method for finding the common element of the set $F(T) \cap \Omega^{*} \cap E P(F)$.

Recently, Censor et al. [4] introduced a new variational inequality problem which we call the split variational inequality problem (SVIP). Let $H_{1}$ and $H_{2}$ be two real Hilbert spaces. Given operators $f: H_{1} \rightarrow H_{1}$ and $g: H_{2} \rightarrow H_{2}$, a bounded linear operator $A: H_{1} \rightarrow H_{2}$, and nonempty, closed and convex subsets $C \subseteq H_{1}$ and $Q \subseteq H_{2}$, the SVIP is formulated as follows: Find a point $x^{*} \in C$ such that

$$
\left\langle f\left(x^{*}\right), x-x^{*}\right\rangle \geq 0 \quad \text { for all } x \in C
$$

and such that

$$
y^{*}=A x^{*} \in Q \quad \text { solves } \quad\left\langle g\left(y^{*}\right), y-y^{*}\right\rangle \geq 0 \quad \text { for all } y \in Q .
$$

In [22], Moudafi introduced an iterative method which can be regarded as an extension of the method given by Censor et al. [4] for the following split monotone variational inclusions:

$$
\text { Find } x^{*} \in H_{1} \text { such that } 0 \in f\left(x^{*}\right)+B_{1}\left(x^{*}\right)
$$

and such that

$$
y^{*}=A x^{*} \in H_{2} \quad \text { solves } \quad 0 \in g\left(y^{*}\right)+B_{2}\left(y^{*}\right),
$$

where $B_{i}: H_{i} \rightarrow 2^{H_{i}}$ is a set-valued mapping for $i=1,2$. Later Byrne et al. [3] generalized and extended the work of Censor et al. [4] and Moudafi [22].

Very recently, Kazmi and Rivzi [13] studied the following pair of equilibrium problems called a split equilibrium problem: Let $F_{1}: C \times C \rightarrow R$ and $F_{2}: Q \times Q \rightarrow R$ be nonlinear bifunctions and $A: H_{1} \rightarrow H_{2}$ be a bounded linear operator, then the split equilibrium problem (SEP) is to find $x^{*} \in C$ such that

$$
F_{1}\left(x^{*}, x\right) \geq 0, \quad \forall x \in C
$$

and such that

$$
y^{*}=A x^{*} \in Q \quad \text { solves } \quad F_{2}\left(y^{*}, y\right) \geq 0, \quad \forall y \in Q .
$$

The solution set of SEP (9)-(10) is denoted by $\Lambda=\left\{p \in E P\left(F_{1}\right): A p \in E P\left(F_{2}\right)\right\}$. 
Let $S: C \rightarrow H$ be a nonexpansive mapping. The following problem is called a hierarchical fixed point problem: Find $x \in F(T)$ such that

$$
\langle x-S x, y-x\rangle \geq 0, \quad \forall y \in F(T)
$$

It is known that the hierarchical fixed point problem (11) links with some monotone variational inequalities and convex programming problems; see [11,27]. Various methods have been proposed to solve the hierarchical fixed point problem; see Moudafi [21], Mainge and Moudafi in [15], Marino and Xu in [17] and Cianciaruso et al. [5]. In 2010, Yao et al. [27] introduced the following strong convergence iterative algorithm to solve problem (11):

$$
\begin{aligned}
& y_{n}=\beta_{n} S x_{n}+\left(1-\beta_{n}\right) x_{n}, \\
& x_{n+1}=P_{C}\left[\alpha_{n} f\left(x_{n}\right)+\left(1-\alpha_{n}\right) T y_{n}\right], \quad \forall n \geq 0,
\end{aligned}
$$

where $f: C \rightarrow H$ is a contraction mapping and $\left\{\alpha_{n}\right\}$ and $\left\{\beta_{n}\right\}$ are two sequences in $(0,1)$. Under some certain restrictions on parameters, Yao et al. proved that the sequence $\left\{x_{n}\right\}$ generated by (12) converges strongly to $z \in F(T)$, which is the unique solution of the following variational inequality:

$$
\langle(I-f) z, y-z\rangle \geq 0, \quad \forall y \in F(T) .
$$

By changing the restrictions on parameters, the authors obtained another result on the iterative scheme (12), the sequence $\left\{x_{n}\right\}$ generated by (12) converges strongly to a point $z \in F(T)$, which is the unique solution of the following variational inequality:

$$
\left\langle\frac{1}{\tau}(I-f) z+(I-S) z, y-z\right\rangle \geq 0, \quad \forall y \in F(T) .
$$

Let $S: C \rightarrow H$ be a nonexpansive mapping and $\left\{T_{i}\right\}_{i=1}^{\infty}: C \rightarrow C$ be a countable family of nonexpansive mappings. In 2011, Gu et al. [11] introduced the following iterative algorithm:

$$
\begin{aligned}
& y_{n}=P_{C}\left[\beta_{n} S x_{n}+\left(1-\beta_{n}\right) x_{n}\right], \\
& x_{n+1}=P_{C}\left[\alpha_{n} f\left(x_{n}\right)+\sum_{i=1}^{n}\left(\alpha_{i-1}-\alpha_{i}\right) T_{i} y_{n}\right], \quad \forall n \geq 1,
\end{aligned}
$$

where $\alpha_{0}=1,\left\{\alpha_{n}\right\}$ is a strictly decreasing sequence in $(0,1)$ and $\left\{\beta_{n}\right\}$ is a sequence in $(0,1)$. Under some certain conditions on parameters, Gu et al. proved that the sequence $\left\{x_{n}\right\}$ generated by (15) converges strongly to $z \in \bigcap_{i=1}^{\infty} F\left(T_{i}\right)$, which is the unique solution of one of variational inequalities (13) and (14).

In this paper, motivated by the work of Censor et al. [4], Moudafi [22], Byrne et al. [3] Kazmi and Rivzi [13], Yao et al. [27] and Gu et al. [11] and by the recent work going on in this direction, we give an iterative method for finding an approximate element of the common set of solutions of (1), (9)-(10) and (11) for a strictly pseudo-contraction mapping in a real Hilbert space. We establish a strong convergence theorem based on this method. The presented method improves and generalizes many known results for solving equilibrium problems, variational inequality problems and hierarchical fixed point problems; see, e.g., $[5,11,15,27]$ and relevant references cited therein. 


\section{Preliminaries}

In this section, we list some fundamental lemmas that are useful in the consequent analysis. The first lemma provides some basic properties of the projection of $H$ onto $C$.

Lemma 2.1 Let $P_{C}$ denote the projection of $H$ onto $C$. Then we have the following inequalities,

$$
\begin{aligned}
& \left\langle z-P_{C}[z], P_{C}[z]-v\right\rangle \geq 0, \quad \forall z \in H, v \in C ; \\
& \left\langle u-v, P_{C}[u]-P_{C}[v]\right\rangle \geq\left\|P_{C}[u]-P_{C}[v]\right\|^{2}, \quad \forall u, v \in H ; \\
& \left\|P_{C}[u]-P_{C}[v]\right\| \leq\|u-v\|, \quad \forall u, v \in H ; \\
& \left\|u-P_{C}[z]\right\|^{2} \leq\|z-u\|^{2}-\left\|z-P_{C}[z]\right\|^{2}, \quad \forall z \in H, u \in C .
\end{aligned}
$$

Assumption 2.1 [2] Let $F: C \times C \rightarrow \mathbb{R}$ be a bifunction satisfying the following assumptions:

(i) $F(x, x)=0, \forall x \in C$;

(ii) $F$ is monotone, i.e., $F(x, y)+F(y, x) \leq 0, \forall x, y \in C$;

(iii) For each $x, y, z \in C, \lim _{t \rightarrow 0} F(t z+(1-t) x, y) \leq F(x, y)$;

(iv) For each $x \in C, y \rightarrow F(x, y)$ is convex and lower semicontinuous;

(v) Fixed $r>0$ and $z \in C$, there exists a bounded subset $K$ of $H_{1}$ and $x \in C \cap K$ such that

$$
F(y, x)+\frac{1}{r}\langle y-x, x-z\rangle \geq 0, \quad \forall y \in C \backslash K .
$$

Lemma 2.2 [8] Assume that $F_{1}: C \times C \rightarrow \mathbb{R}$ satisfies Assumption 2.1. For $r>0$ and $\forall x \in$ $H_{1}$, define a mapping $T_{r}^{F_{1}}: H_{1} \rightarrow C$ as follows:

$$
T_{r}^{F_{1}}(x)=\left\{z \in C: F_{1}(z, y)+\frac{1}{r}\langle y-z, z-x\rangle \geq 0, \forall y \in C\right\} .
$$

Then the following hold:

(i) $T_{r}^{F_{1}}$ is nonempty and single-valued;

(ii) $T_{r}^{F_{1}}$ is firmly nonexpansive, i.e.,

$$
\left\|T_{r}^{F_{1}}(x)-T_{r}^{F_{1}}(y)\right\|^{2} \leq\left\langle T_{r}^{F_{1}}(x)-T_{r}^{F_{1}}(y), x-y\right\rangle, \quad \forall x, y \in H_{1} ;
$$

(iii) $F\left(T_{r}^{F_{1}}\right)=E P\left(F_{1}\right)$;

(iv) $E P\left(F_{1}\right)$ is closed and convex.

Assume that $F_{2}: Q \times Q \rightarrow \mathbb{R}$ satisfies Assumption 2.1. For $s>0$ and $\forall u \in H_{2}$, define a mapping $T_{s}^{F_{2}}: H_{2} \rightarrow Q$ as follows:

$$
T_{s}^{F_{2}}(u)=\left\{v \in Q: F_{2}(v, w)+\frac{1}{s}\langle w-v, v-u\rangle \geq 0, \forall w \in Q\right\} .
$$

Then $T_{s}^{F_{2}}$ satisfies conditions (i)-(iv) of Lemma 2.2. $F\left(T_{s}^{F_{2}}\right)=E P\left(F_{2}, Q\right)$, where $E P\left(F_{2}, Q\right)$ is the solution set of the following equilibrium problem:

$$
\text { Find } y^{*} \in Q \text { such that } F_{2}\left(y^{*}, y\right) \geq 0, \quad \forall y \in Q \text {. }
$$


Lemma 2.3 [6] Assume that $F_{1}: C \times C \rightarrow \mathbb{R}$ satisfies Assumption 2.1, and let $T_{r}^{F_{1}}$ be defined as in Lemma 2.2. Let $x, y \in H_{1}$ and $r_{1}, r_{2}>0$. Then

$$
\left\|T_{r_{2}}^{F_{1}}(y)-T_{r_{1}}^{F_{1}}(x)\right\| \leq\|y-x\|+\left|\frac{r_{2}-r_{1}}{r_{2}}\right|\left\|T_{r_{2}}^{F_{1}}(y)-y\right\| .
$$

Lemma 2.4 [28] Let $C$ be a nonempty closed convex subset of a real Hilbert space $H$. If $T: C \rightarrow C$ is a k-strict pseudo-contraction, then:

(i) The mapping $I-T$ is demiclosed at 0 , i.e., if $\left\{x_{n}\right\}$ is a sequence in $C$ weakly converging to $x$ and if $\left\{(I-T) x_{n}\right\}$ converges strongly to 0 , then $(I-T) x=0$;

(ii) The set $F(T)$ of $T$ is closed and convex so that the projection $P_{F(T)}$ is well defined.

Lemma 2.5 [16] Let $H$ be a real Hilbert space. Then the following inequality holds:

$$
\|x+y\|^{2} \leq\|x\|^{2}+2\langle y, x+y\rangle, \quad \forall x, y \in H .
$$

Lemma 2.6 [26] Assume that $\left\{a_{n}\right\}$ is a sequence of nonnegative real numbers such that

$$
a_{n+1} \leq\left(1-\gamma_{n}\right) a_{n}+\delta_{n}
$$

where $\left\{\gamma_{n}\right\}$ is a sequence in $(0,1)$ and $\left\{\delta_{n}\right\}$ is a sequence such that

(1) $\sum_{n=1}^{\infty} \gamma_{n}=\infty$;

(2) $\lim \sup _{n \rightarrow \infty} \delta_{n} / \gamma_{n} \leq 0$ or $\sum_{n=1}^{\infty}\left|\delta_{n}\right|<\infty$.

Then $\lim _{n \rightarrow \infty} a_{n}=0$.

Lemma 2.7 [1] Let $C$ be a closed convex subset of $H$. Let $\left\{x_{n}\right\}$ be a bounded sequence in $H$. Assume that

(i) the weak $w$-limit set $w_{w}\left(x_{n}\right) \subset C$, where $w_{w}\left(x_{n}\right)=\left\{x: x_{n_{i}} \rightarrow x\right\}$;

(ii) for each $z \in C, \lim _{n \rightarrow \infty}\left\|x_{n}-z\right\|$ exists.

Then $\left\{x_{n}\right\}$ is weakly convergent to a point in $C$.

Lemma 2.8 [29] Let $H$ be a Hilbert space, $C$ be a closed and convex subset of $H$, and $T: C \rightarrow C$ be a k-strict pseudo-contraction mapping. Define a mapping $V: C \rightarrow H$ by $V x=\lambda x+(1-\lambda) T x, \forall x \in C$. Then, as $k \leq \lambda<1, V$ is a nonexpansive mapping such that $F(V)=F(T)$.

Lemma 2.9 [11] Let $H$ be a Hilbert space, $C$ be a closed and convex subset of $H$, and $T$ : $C \rightarrow C$ be a nonexpansive mapping such that $F(T) \neq \emptyset$. Then

$$
\|T x-x\|^{2} \leq 2\left(x-T x, x-x^{\prime}\right\rangle, \quad \forall x^{\prime} \in F(T), \forall x \in C .
$$

\section{The proposed method and some properties}

In this section, we suggest and analyze our method for finding common solutions of the variational inequality (1), the split equilibrium problem (9)-(10) and the hierarchical fixed point problem (11).

Let $H_{1}$ and $H_{2}$ be two real Hilbert spaces and $C \subseteq H_{1}$ and $Q \subseteq H_{2}$ be nonempty closed convex subsets of Hilbert spaces $H_{1}$ and $H_{2}$, respectively. Let $A: H_{1} \rightarrow H_{2}$ be a bounded 
linear operator. Let $D: C \rightarrow H_{1}$ be an $\alpha$-inverse strongly monotone mapping. Assume that $F_{1}: C \times C \rightarrow \mathbb{R}$ and $F_{2}: Q \times Q \rightarrow \mathbb{R}$ are the bifunctions satisfying Assumption 2.1 and $F_{2}$ is upper semicontinuous in the first argument. Let $S: C \rightarrow H_{1}$ be a nonexpansive mapping and $\left\{T_{i}\right\}_{i=1}^{\infty}: C \rightarrow C$ be a countable family of $k_{i}$-strict pseudo-contraction mappings such that $F(T) \cap \Omega^{*} \cap \Lambda \neq \emptyset$, where $F(T)=\bigcap_{i=1}^{\infty} F\left(T_{i}\right)$. Let $f$ be a $\rho$-contraction mapping.

Algorithm 3.1 For a given $x_{0} \in C$ arbitrarily, let the iterative sequences $\left\{u_{n}\right\},\left\{x_{n}\right\},\left\{y_{n}\right\}$ and $\left\{z_{n}\right\}$ be generated by

$$
\begin{aligned}
& u_{n}=T_{r_{n}}^{F_{1}}\left(x_{n}+\gamma A^{*}\left(T_{r_{n}}^{F_{2}}-I\right) A x_{n}\right) ; \\
& z_{n}=P_{C}\left[u_{n}-\lambda_{n} D u_{n}\right] ; \\
& y_{n}=P_{C}\left[\beta_{n} S x_{n}+\left(1-\beta_{n}\right) z_{n}\right] ; \\
& x_{n+1}=P_{C}\left[\alpha_{n} f\left(x_{n}\right)+\sum_{i=1}^{n}\left(\alpha_{i-1}-\alpha_{i}\right) V_{i} y_{n}\right], \quad \forall n \geq 0,
\end{aligned}
$$

where $V_{i}=k_{i} I+\left(1-k_{i}\right) T_{i}, 0 \leq k_{i}<1,\left\{r_{n}\right\} \subset(0, \infty),\left\{\lambda_{n}\right\} \subset(0,2 \alpha)$ and $\gamma \in(0,1 / L), L$ is the spectral radius of the operator $A^{*} A$ and $A^{*}$ is the adjoint of $A$ and $\alpha_{0}=1,\left\{\alpha_{n}\right\}$ is a strictly decreasing sequence in $(0,1)$ and $\left\{\beta_{n}\right\}$ is a sequence in $(0,1)$ satisfying the following conditions:

(a) $\lim _{n \rightarrow \infty} \alpha_{n}=0$ and $\sum_{n=1}^{\infty} \alpha_{n}=\infty$,

(b) $\lim _{n \rightarrow \infty}\left(\beta_{n} / \alpha_{n}\right)=0$,

(c) $\sum_{n=1}^{\infty}\left|\alpha_{n-1}-\alpha_{n}\right|<\infty$ and $\sum_{n=1}^{\infty}\left|\beta_{n-1}-\beta_{n}\right|<\infty$,

(d) $\liminf _{n \rightarrow \infty} r_{n}>0$ and $\sum_{n=1}^{\infty}\left|r_{n-1}-r_{n}\right|<\infty$,

(e) $\liminf _{n \rightarrow \infty} \lambda_{n}<\lim \sup _{n \rightarrow \infty} \lambda_{n}<2 \alpha$ and $\sum_{n=1}^{\infty}\left|\lambda_{n-1}-\lambda_{n}\right|<\infty$.

Lemma 3.1 Let $x^{*} \in F(T) \cap \Omega^{*} \cap \Lambda$. Then $\left\{x_{n}\right\},\left\{u_{n}\right\},\left\{z_{n}\right\}$ and $\left\{y_{n}\right\}$ are bounded.

Proof First, we show that the mapping $\left(I-\lambda_{n} D\right)$ is nonexpansive. For any $x, y \in C$,

$$
\begin{aligned}
\left\|\left(I-\lambda_{n} D\right) x-\left(I-\lambda_{n} D\right) y\right\|^{2} & =\left\|(x-y)-\lambda_{n}(D x-D y)\right\|^{2} \\
& =\|x-y\|^{2}-2 \lambda_{n}\langle x-y, D x-D y\rangle+\lambda_{n}^{2}\|D x-D y\|^{2} \\
& \leq\|x-y\|^{2}-\lambda_{n}\left(2 \alpha-\lambda_{n}\right)\|D x-D y\|^{2} \\
& \leq\|x-y\|^{2} .
\end{aligned}
$$

Let $x^{*} \in F(T) \cap \Omega^{*} \cap \Lambda$, we have $x^{*}=T_{r_{n}}^{F_{1}}\left(x^{*}\right)$ and $A x^{*}=T_{r_{n}}^{F_{2}}\left(A x^{*}\right)$. Then

$$
\begin{aligned}
\left\|u_{n}-x^{*}\right\|^{2}= & \left\|T_{r_{n}}^{F_{1}}\left(x_{n}+\gamma A^{*}\left(T_{r_{n}}^{F_{2}}-I\right) A x_{n}\right)-x^{*}\right\|^{2} \\
= & \left\|T_{r_{n}}^{F_{1}}\left(x_{n}+\gamma A^{*}\left(T_{r_{n}}^{F_{2}}-I\right) A x_{n}\right)-T_{r_{n}}^{F_{1}}\left(x^{*}\right)\right\|^{2} \\
\leq & \left\|x_{n}+\gamma A^{*}\left(T_{r_{n}}^{F_{2}}-I\right) A x_{n}-x^{*}\right\|^{2} \\
= & \left\|x_{n}-x^{*}\right\|^{2}+\gamma^{2}\left\|A^{*}\left(T_{r_{n}}^{F_{2}}-I\right) A x_{n}\right\|^{2}+2 \gamma\left(x_{n}-x^{*}, A^{*}\left(T_{r_{n}}^{F_{2}}-I\right) A x_{n}\right\rangle \\
= & \left\|x_{n}-x^{*}\right\|^{2}+\gamma^{2}\left\langle\left(T_{r_{n}}^{F_{2}}-I\right) A x_{n}, A A^{*}\left(T_{r_{n}}^{F_{2}}-I\right) A x_{n}\right\rangle \\
& +2 \gamma\left(x_{n}-x^{*}, A^{*}\left(T_{r_{n}}^{F_{2}}-I\right) A x_{n}\right\rangle .
\end{aligned}
$$


From the definition of $L$, it follows that

$$
\begin{aligned}
\gamma^{2}\left\langle\left(T_{r_{n}}^{F_{2}}-I\right) A x_{n}, A A^{*}\left(T_{r_{n}}^{F_{2}}-I\right) A x_{n}\right\rangle & \leq L \gamma^{2}\left\langle\left(T_{r_{n}}^{F_{2}}-I\right) A x_{n},\left(T_{r_{n}}^{F_{2}}-I\right) A x_{n}\right\rangle \\
& =L \gamma^{2}\left\|\left(T_{r_{n}}^{F_{2}}-I\right) A x_{n}\right\|^{2}
\end{aligned}
$$

It follows from (3) that

$$
\begin{aligned}
& 2 \gamma\left\langle x_{n}-x^{*}, A^{*}\left(T_{r_{n}}^{F_{2}}-I\right) A x_{n}\right\rangle \\
& \quad=2 \gamma\left\langle A\left(x_{n}-x^{*}\right),\left(T_{r_{n}}^{F_{2}}-I\right) A x_{n}\right\rangle \\
& \quad=2 \gamma\left\langle A\left(x_{n}-x^{*}\right)+\left(T_{r_{n}}^{F_{2}}-I\right) A x_{n}-\left(T_{r_{n}}^{F_{2}}-I\right) A x_{n},\left(T_{r_{n}}^{F_{2}}-I\right) A x_{n}\right\rangle \\
& \quad=2 \gamma\left(\left\langle T_{r_{n}}^{F_{2}} A x_{n}-A x^{*},\left(T_{r_{n}}^{F_{2}}-I\right) A x_{n}\right\rangle-\left\|\left(T_{r_{n}}^{F_{2}}-I\right) A x_{n}\right\|^{2}\right) \\
& \quad \leq 2 \gamma\left(\frac{1}{2}\left\|\left(T_{r_{n}}^{F_{2}}-I\right) A x_{n}\right\|^{2}-\left\|\left(T_{r_{n}}^{F_{2}}-I\right) A x_{n}\right\|^{2}\right) \\
& \quad=-\gamma\left\|\left(T_{r_{n}}^{F_{2}}-I\right) A x_{n}\right\|^{2} .
\end{aligned}
$$

Applying (23) and (22) to (21) and from the definition of $\gamma$, we get

$$
\left\|u_{n}-x^{*}\right\|^{2} \leq\left\|x_{n}-x^{*}\right\|^{2}+\gamma(L \gamma-1)\left\|\left(T_{r_{n}}^{F_{2}}-I\right) A x_{n}\right\|^{2} \leq\left\|x_{n}-x^{*}\right\|^{2} .
$$

Since the mapping $D$ is $\alpha$-inverse strongly monotone, we have

$$
\begin{aligned}
\left\|z_{n}-x^{*}\right\|^{2} & =\left\|P_{C}\left[u_{n}-\lambda_{n} D u_{n}\right]-P_{C}\left[x^{*}-\lambda_{n} D x^{*}\right]\right\|^{2} \\
& \leq\left\|u_{n}-x^{*}-\lambda_{n}\left(D u_{n}-D x^{*}\right)\right\|^{2} \\
& \leq\left\|u_{n}-x^{*}\right\|^{2}-\lambda_{n}\left(2 \alpha-\lambda_{n}\right)\left\|D u_{n}-D x^{*}\right\|^{2} \\
& \leq\left\|u_{n}-x^{*}\right\|^{2} \\
& \leq\left\|x_{n}-x^{*}\right\|^{2} .
\end{aligned}
$$

Next, we prove that the sequence $\left\{x_{n}\right\}$ is bounded, without loss of generality, we can assume that $\beta_{n} \leq \alpha_{n}$ for all $n \geq 1$. From Lemma 2.8, we have $V_{i}$ is a nonexpansive mapping and $V_{i} x^{*}=x^{*}$. Since $\sum_{i=1}^{n}\left(\alpha_{i-1}-\alpha_{i}\right)=1-\alpha_{n}$, we get

$$
\begin{aligned}
\left\|x_{n+1}-x^{*}\right\| & =\left\|P_{C}\left[\alpha_{n} f\left(x_{n}\right)+\sum_{i=1}^{n}\left(\alpha_{i-1}-\alpha_{i}\right) V_{i} y_{n}\right]-x^{*}\right\| \\
& \leq\left\|\alpha_{n} f\left(x_{n}\right)+\sum_{i=1}^{n}\left(\alpha_{i-1}-\alpha_{i}\right) V_{i} y_{n}-x^{*}\right\| \\
& =\left\|\alpha_{n} f\left(x_{n}\right)+\sum_{i=1}^{n}\left(\alpha_{i-1}-\alpha_{i}\right) V_{i} y_{n}-\alpha_{n} x^{*}-\sum_{i=1}^{n}\left(\alpha_{i-1}-\alpha_{i}\right) V_{i} x^{*}\right\| \\
& \leq \alpha_{n}\left\|f\left(x_{n}\right)-f\left(x^{*}\right)\right\|+\alpha_{n}\left\|f\left(x^{*}\right)-x^{*}\right\|+\sum_{i=1}^{n}\left(\alpha_{i-1}-\alpha_{i}\right)\left\|V_{i} y_{n}-V_{i} x^{*}\right\| \\
& \leq \alpha_{n}\left\|f\left(x_{n}\right)-f\left(x^{*}\right)\right\|+\alpha_{n}\left\|f\left(x^{*}\right)-x^{*}\right\|+\sum_{i=1}^{n}\left(\alpha_{i-1}-\alpha_{i}\right)\left\|y_{n}-x^{*}\right\|
\end{aligned}
$$




$$
\begin{aligned}
= & \alpha_{n}\left\|f\left(x_{n}\right)-f\left(x^{*}\right)\right\|+\alpha_{n}\left\|f\left(x^{*}\right)-x^{*}\right\|+\left(1-\alpha_{n}\right)\left\|\beta_{n} S x_{n}+\left(1-\beta_{n}\right) z_{n}-x^{*}\right\| \\
\leq & \alpha_{n}\left\|f\left(x_{n}\right)-f\left(x^{*}\right)\right\|+\alpha_{n}\left\|f\left(x^{*}\right)-x^{*}\right\| \\
& +\left(1-\alpha_{n}\right)\left(\beta_{n}\left\|S x_{n}-S x^{*}\right\|+\beta_{n}\left\|S x^{*}-x^{*}\right\|+\left(1-\beta_{n}\right)\left\|z_{n}-x^{*}\right\|\right) \\
\leq & \alpha_{n} \rho\left\|x_{n}-x^{*}\right\|+\alpha_{n}\left\|f\left(x^{*}\right)-x^{*}\right\| \\
& +\left(1-\alpha_{n}\right)\left(\beta_{n}\left\|x_{n}-x^{*}\right\|+\beta_{n}\left\|S x^{*}-x^{*}\right\|+\left(1-\beta_{n}\right)\left\|x_{n}-x^{*}\right\|\right) \\
= & \left(1-\alpha_{n}(1-\rho)\right)\left\|x_{n}-x^{*}\right\|+\alpha_{n}\left\|f\left(x^{*}\right)-x^{*}\right\|+\left(1-\alpha_{n}\right) \beta_{n}\left\|S x^{*}-x^{*}\right\| \\
\leq & \left(1-\alpha_{n}(1-\rho)\right)\left\|x_{n}-x^{*}\right\|+\alpha_{n}\left\|f\left(x^{*}\right)-x^{*}\right\|+\beta_{n}\left\|S x^{*}-x^{*}\right\| \\
\leq & \left(1-\alpha_{n}(1-\rho)\right)\left\|x_{n}-x^{*}\right\|+\alpha_{n}\left(\left\|f\left(x^{*}\right)-x^{*}\right\|+\left\|S x^{*}-x^{*}\right\|\right) \\
= & \left(1-\alpha_{n}(1-\rho)\right)\left\|x_{n}-x^{*}\right\|+\frac{\alpha_{n}(1-\rho)}{1-\rho}\left(\left\|f\left(x^{*}\right)-x^{*}\right\|+\left\|S x^{*}-x^{*}\right\|\right) \\
\leq & \max \left\{\left\|x_{n}-x^{*}\right\|, \frac{1}{1-\rho}\left(\left\|f\left(x^{*}\right)-x^{*}\right\|+\left\|S x^{*}-x^{*}\right\|\right)\right\} .
\end{aligned}
$$

By induction on $n$, we obtain $\left\|x_{n}-x^{*}\right\| \leq \max \left\{\left\|x_{0}-x^{*}\right\|, \frac{1}{1-\rho}\left(\left\|f\left(x^{*}\right)-x^{*}\right\|+\left\|S x^{*}-x^{*}\right\|\right)\right\}$, for $n \geq 0$ and $x_{0} \in C$. Hence $\left\{x_{n}\right\}$ is bounded and consequently, we deduce that $\left\{u_{n}\right\},\left\{z_{n}\right\}$ and $\left\{y_{n}\right\}$ are bounded.

Lemma 3.2 Let $x^{*} \in F(T) \cap \Omega^{*} \cap \Lambda$ and $\left\{x_{n}\right\}$ be the sequence generated by Algorithm 3.1. Then we have

(a) $\lim _{n \rightarrow \infty}\left\|x_{n+1}-x_{n}\right\|=0$;

(b) The weak w-limit set $w_{w}\left(x_{n}\right) \subset F(T)\left(w_{w}\left(x_{n}\right)=\left\{x: x_{n_{i}} \rightarrow x\right\}\right)$.

Proof From the nonexpansivity of the mapping $\left(I-\lambda_{n} D\right)$ and $P_{C}$, we have

$$
\begin{aligned}
\left\|z_{n}-z_{n-1}\right\| & \leq\left\|\left(u_{n}-\lambda_{n} D u_{n}\right)-\left(u_{n-1}-\lambda_{n-1} D u_{n-1}\right)\right\| \\
& =\left\|\left(u_{n}-u_{n-1}\right)-\lambda_{n}\left(D u_{n}-D u_{n-1}\right)-\left(\lambda_{n}-\lambda_{n-1}\right) D u_{n-1}\right\| \\
& \leq\left\|\left(u_{n}-u_{n-1}\right)-\lambda_{n}\left(D u_{n}-D u_{n-1}\right)\right\|+\left|\lambda_{n}-\lambda_{n-1}\right|\left\|D u_{n-1}\right\| \\
& \leq\left\|u_{n}-u_{n-1}\right\|+\left|\lambda_{n}-\lambda_{n-1}\right|\left\|D u_{n-1}\right\| .
\end{aligned}
$$

Next, we estimate

$$
\begin{aligned}
& \left\|y_{n}-y_{n-1}\right\| \\
& \quad \leq\left\|\beta_{n} S x_{n}+\left(1-\beta_{n}\right) z_{n}-\left(\beta_{n-1} S x_{n-1}+\left(1-\beta_{n-1}\right) z_{n-1}\right)\right\| \\
& \quad=\left\|\beta_{n}\left(S x_{n}-S x_{n-1}\right)+\left(\beta_{n}-\beta_{n-1}\right) S x_{n-1}+\left(1-\beta_{n}\right)\left(z_{n}-z_{n-1}\right)+\left(\beta_{n-1}-\beta_{n}\right) z_{n-1}\right\| \\
& \quad \leq \beta_{n}\left\|x_{n}-x_{n-1}\right\|+\left(1-\beta_{n}\right)\left\|z_{n}-z_{n-1}\right\|+\left|\beta_{n}-\beta_{n-1}\right|\left(\left\|S x_{n-1}\right\|+\left\|z_{n-1}\right\|\right) .
\end{aligned}
$$

It follows from (27) and (28) that

$$
\begin{aligned}
\left\|y_{n}-y_{n-1}\right\| \leq & \beta_{n}\left\|x_{n}-x_{n-1}\right\|+\left(1-\beta_{n}\right)\left\{\left\|u_{n}-u_{n-1}\right\|+\left|\lambda_{n}-\lambda_{n-1}\right|\left\|D u_{n-1}\right\|\right\} \\
& +\left|\beta_{n}-\beta_{n-1}\right|\left(\left\|S x_{n-1}\right\|+\left\|z_{n-1}\right\|\right) .
\end{aligned}
$$


On the other hand, $u_{n}=T_{r_{n}}^{F_{1}}\left(x_{n}+\gamma A^{*}\left(T_{r_{n}}^{F_{2}}-I\right) A x_{n}\right)$ and $u_{n-1}=T_{r_{n-1}}^{F_{1}}\left(x_{n-1}+\gamma A^{*}\left(T_{r_{n-1}}^{F_{2}}-\right.\right.$ I) $\left.A x_{n-1}\right)$. It follows from Lemma 2.3 that

$$
\begin{aligned}
& \left\|u_{n}-u_{n-1}\right\| \\
& \leq\left\|x_{n}-x_{n-1}+\gamma\left(A^{*}\left(T_{r_{n}}^{F_{2}}-I\right) A x_{n}-A^{*}\left(T_{r_{n-1}}^{F_{2}}-I\right) A x_{n-1}\right)\right\| \\
& +\left|1-\frac{r_{n-1}}{r_{n}}\right|\left\|T_{r_{n}}^{F_{1}}\left(x_{n}+\gamma A^{*}\left(T_{r_{n}}^{F_{2}}-I\right) A x_{n}\right)-\left(x_{n}+\gamma A^{*}\left(T_{r_{n}}^{F_{2}}-I\right) A x_{n}\right)\right\| \\
& \leq\left\|x_{n}-x_{n-1}-\gamma A^{*} A\left(x_{n}-x_{n-1}\right)\right\|+\gamma\|A\|\left\|T_{r_{n}}^{F_{2}} A x_{n}-T_{r_{n-1}}^{F_{2}} A x_{n-1}\right\| \\
& +\left|1-\frac{r_{n-1}}{r_{n}}\right|\left\|T_{r_{n}}^{F_{1}}\left(x_{n}+\gamma A^{*}\left(T_{r_{n}}^{F_{2}}-I\right) A x_{n}\right)-\left(x_{n}+\gamma A^{*}\left(T_{r_{n}}^{F_{2}}-I\right) A x_{n}\right)\right\| \\
& \leq\left(\left\|x_{n}-x_{n-1}\right\|^{2}-2 \gamma\left\|A\left(x_{n}-x_{n-1}\right)\right\|^{2}+\gamma^{2}\|A\|^{4}\left\|x_{n}-x_{n-1}\right\|^{2}\right)^{\frac{1}{2}} \\
& +\gamma\|A\|\left(\left\|A\left(x_{n}-x_{n-1}\right)\right\|+\left|1-\frac{r_{n-1}}{r_{n}}\right|\left\|T_{r_{n}}^{F_{2}} A x_{n}-A x_{n}\right\|\right) \\
& +\left|1-\frac{r_{n-1}}{r_{n}}\right|\left\|T_{r_{n}}^{F_{1}}\left(x_{n}+\gamma A^{*}\left(T_{r_{n}}^{F_{2}}-I\right) A x_{n}\right)-\left(x_{n}+\gamma A^{*}\left(T_{r_{n}}^{F_{2}}-I\right) A x_{n}\right)\right\| \\
& \leq\left(1-2 \gamma\|A\|^{2}+\gamma^{2}\|A\|^{4}\right)^{\frac{1}{2}}\left\|x_{n}-x_{n-1}\right\|+\gamma\|A\|^{2}\left\|x_{n}-x_{n-1}\right\| \\
& +\gamma\|A\|\left|1-\frac{r_{n-1}}{r_{n}}\right|\left\|T_{r_{n}}^{F_{2}} A x_{n}-A x_{n}\right\| \\
& +\left|1-\frac{r_{n-1}}{r_{n}}\right|\left\|T_{r_{n}}^{F_{1}}\left(x_{n}+\gamma A^{*}\left(T_{r_{n}}^{F_{2}}-I\right) A x_{n}\right)-\left(x_{n}+\gamma A^{*}\left(T_{r_{n}}^{F_{2}}-I\right) A x_{n}\right)\right\| \\
& =\left(1-\gamma\|A\|^{2}\right)\left\|x_{n}-x_{n-1}\right\|+\gamma\|A\|^{2}\left\|x_{n}-x_{n-1}\right\|+\gamma\|A\|\left|1-\frac{r_{n-1}}{r_{n}}\right|\left\|T_{r_{n}}^{F_{2}} A x_{n}-A x_{n}\right\| \\
& +\left|1-\frac{r_{n-1}}{r_{n}}\right|\left\|T_{r_{n}}^{F_{1}}\left(x_{n}+\gamma A^{*}\left(T_{r_{n}}^{F_{2}}-I\right) A x_{n}\right)-\left(x_{n}+\gamma A^{*}\left(T_{r_{n}}^{F_{2}}-I\right) A x_{n}\right)\right\| \\
& =\left\|x_{n}-x_{n-1}\right\|+\gamma\|A\|\left|1-\frac{r_{n-1}}{r_{n}}\right|\left\|T_{r_{n}}^{F_{2}} A x_{n}-A x_{n}\right\| \\
& +\left|1-\frac{r_{n-1}}{r_{n}}\right|\left\|T_{r_{n}}^{F_{1}}\left(x_{n}+\gamma A^{*}\left(T_{r_{n}}^{F_{2}}-I\right) A x_{n}\right)-\left(x_{n}+\gamma A^{*}\left(T_{r_{n}}^{F_{2}}-I\right) A x_{n}\right)\right\| \\
& =\left\|x_{n}-x_{n-1}\right\|+\left|\frac{r_{n}-r_{n-1}}{r_{n}}\right|\left(\gamma\|A\| \sigma_{n}+\chi_{n}\right) \text {, }
\end{aligned}
$$

where $\sigma_{n}:=\left\|T_{r_{n}}^{F_{2}} A x_{n}-A x_{n}\right\|$ and $\chi_{n}:=\| T_{r_{n}}^{F_{1}}\left(x_{n}+\gamma A^{*}\left(T_{r_{n}}^{F_{2}}-I\right) A x_{n}\right)-\left(x_{n}+\gamma A^{*}\left(T_{r_{n}}^{F_{2}}-\right.\right.$ I) $\left.A x_{n}\right) \|$. Without loss of generality, let us assume that there exists a real number $\mu$ such that $r_{n}>\mu>0$ for all positive integers $n$. Then we get

$$
\left\|u_{n-1}-u_{n}\right\| \leq\left\|x_{n-1}-x_{n}\right\|+\frac{1}{\mu}\left|r_{n-1}-r_{n}\right|\left(\gamma\|A\| \sigma_{n}+\chi_{n}\right) .
$$

It follows from (29) and (30) that

$$
\begin{aligned}
& \left\|y_{n}-y_{n-1}\right\| \\
& \quad \leq \beta_{n}\left\|x_{n}-x_{n-1}\right\|+\left(1-\beta_{n}\right)\left\{\left\|x_{n}-x_{n-1}\right\|+\frac{1}{\mu}\left|r_{n-1}-r_{n}\right|\left(\gamma\|A\| \sigma_{n}+\chi_{n}\right)\right.
\end{aligned}
$$




$$
\begin{aligned}
& \left.+\left|\lambda_{n}-\lambda_{n-1}\right|\left\|D u_{n-1}\right\|\right\}+\left|\beta_{n}-\beta_{n-1}\right|\left(\left\|S x_{n-1}\right\|+\left\|z_{n-1}\right\|\right) \\
= & \left\|x_{n}-x_{n-1}\right\|+\left(1-\beta_{n}\right)\left\{\frac{1}{\mu}\left|r_{n-1}-r_{n}\right|\left(\gamma\|A\| \sigma_{n}+\chi_{n}\right)+\left|\lambda_{n}-\lambda_{n-1}\right|\left\|D u_{n-1}\right\|\right\} \\
& +\left|\beta_{n}-\beta_{n-1}\right|\left(\left\|S x_{n-1}\right\|+\left\|z_{n-1}\right\|\right) .
\end{aligned}
$$

Next, we estimate

$$
\begin{aligned}
\left\|x_{n+1}-x_{n}\right\| & \left\|\alpha_{n} f\left(x_{n}\right)+\sum_{i=1}^{n}\left(\alpha_{i-1}-\alpha_{i}\right) V_{i} y_{n}-\left(\alpha_{n-1} f\left(x_{n-1}\right)+\sum_{i=1}^{n-1}\left(\alpha_{i-1}-\alpha_{i}\right) V_{i} y_{n-1}\right)\right\| \\
= & \| \alpha_{n}\left(f\left(x_{n}\right)-f\left(x_{n-1}\right)\right)+\left(\alpha_{n}-\alpha_{n-1}\right) f\left(x_{n-1}\right)+\sum_{i=1}^{n}\left(\alpha_{i-1}-\alpha_{i}\right)\left(V_{i} y_{n}-V_{i} y_{n-1}\right) \\
& +\left(\alpha_{n-1}-\alpha_{n}\right) V_{n} y_{n-1} \| \\
\leq & \alpha_{n}\left\|f\left(x_{n}\right)-f\left(x_{n-1}\right)\right\|+\sum_{i=1}^{n}\left(\alpha_{i-1}-\alpha_{i}\right)\left\|V_{i} y_{n}-V_{i} y_{n-1}\right\| \\
& +\left|\alpha_{n}-\alpha_{n-1}\right|\left(\left\|f\left(x_{n-1}\right)\right\|+\left\|V_{n} y_{n-1}\right\|\right) \\
\leq & \alpha_{n} \rho\left\|x_{n}-x_{n-1}\right\|+\sum_{i=1}^{n}\left(\alpha_{i-1}-\alpha_{i}\right)\left\|y_{n}-y_{n-1}\right\| \\
& +\left|\alpha_{n}-\alpha_{n-1}\right|\left(\left\|f\left(x_{n-1}\right)\right\|+\left\|V_{n} y_{n-1}\right\|\right) \\
= & \alpha_{n} \rho\left\|x_{n}-x_{n-1}\right\|+\left(1-\alpha_{n}\right)\left\|y_{n}-y_{n-1}\right\| \\
& +\left|\alpha_{n}-\alpha_{n-1}\right|\left(\left\|f\left(x_{n-1}\right)\right\|+\left\|V_{n} y_{n-1}\right\|\right) .
\end{aligned}
$$

From (31) and (32), we have

$$
\begin{aligned}
&\left\|x_{n+1}-x_{n}\right\| \\
& \leq \alpha_{n} \rho\left\|x_{n}-x_{n-1}\right\|+\left(1-\alpha_{n}\right)\left\{\left\|x_{n}-x_{n-1}\right\|\right. \\
&+\left(1-\beta_{n}\right)\left(\frac{1}{\mu}\left|r_{n-1}-r_{n}\right|\left(\gamma\|A\| \sigma_{n}+\chi_{n}\right)+\left|\lambda_{n}-\lambda_{n-1}\right|\left\|D u_{n-1}\right\|\right) \\
&\left.+\left|\beta_{n}-\beta_{n-1}\right|\left(\left\|S x_{n-1}\right\|+\left\|z_{n-1}\right\|\right)\right\}+\left|\alpha_{n}-\alpha_{n-1}\right|\left(\left\|f\left(x_{n-1}\right)\right\|+\left\|V_{n} y_{n-1}\right\|\right) \\
& \leq\left(1-(1-\rho) \alpha_{n}\right)\left\|x_{n}-x_{n-1}\right\|+\frac{1}{\mu}\left|r_{n-1}-r_{n}\right|\left(\gamma\|A\| \sigma_{n}+\chi_{n}\right) \\
&+\left|\lambda_{n}-\lambda_{n-1}\right|\left\|D u_{n-1}\right\|+\left|\beta_{n}-\beta_{n-1}\right|\left(\left\|S x_{n-1}\right\|+\left\|z_{n-1}\right\|\right) \\
&+\left|\alpha_{n}-\alpha_{n-1}\right|\left(\left\|f\left(x_{n-1}\right)\right\|+\left\|V_{n} y_{n-1}\right\|\right) \\
& \leq\left(1-(1-\rho) \alpha_{n}\right)\left\|x_{n}-x_{n-1}\right\| \\
&+M\left(\frac{1}{\mu}\left|r_{n}-r_{n-1}\right|+\left|\lambda_{n}-\lambda_{n-1}\right|+\left|\beta_{n}-\beta_{n-1}\right|+\left|\alpha_{n}-\alpha_{n-1}\right|\right),
\end{aligned}
$$


where

$$
\begin{aligned}
M= & \max \left\{\sup _{n \geq 1}\left(\gamma\|A\| \sigma_{n}+\chi_{n}\right), \sup _{n \geq 1}\left\|D u_{n-1}\right\|, \sup _{n \geq 1}\left(\left\|S x_{n-1}\right\|+\left\|z_{n-1}\right\|\right),\right. \\
& \left.\sup _{n \geq 1}\left(\left\|f\left(x_{n-1}\right)\right\|+\left\|V_{n} y_{n-1}\right\|\right)\right\} .
\end{aligned}
$$

Since $\left\{x_{n}\right\},\left\{u_{n}\right\},\left\{z_{n}\right\}$ and $\left\{y_{n}\right\}$ are bounded, we deduce that $\left\{A x_{n}\right\},\left\{D u_{n-1}\right\},\left\{S x_{n-1}\right\}$, $\left\{f\left(x_{n-1}\right)\right\}$ and $\left\{V_{n} y_{n-1}\right\}$ are bounded. We can conclude that $\sup _{n \geq 1}\left(\gamma\|A\| \sigma_{n}+\chi_{n}\right)<\infty$, $\sup _{n \geq 1}\left\|D u_{n-1}\right\|<\infty, \sup _{n \geq 1}\left(\left\|S x_{n-1}\right\|+\left\|z_{n-1}\right\|\right)<\infty, \sup _{n \geq 1}\left(\left\|f\left(x_{n-1}\right)\right\|+\left\|V_{n} y_{n-1}\right\|\right)<\infty$, and $M<\infty$.

It follows by conditions (a)-(e) of Algorithm 3.1 and Lemma 2.6 that

$$
\lim _{n \rightarrow \infty}\left\|x_{n+1}-x_{n}\right\|=0
$$

Next, we show that $\lim _{n \rightarrow \infty}\left\|u_{n}-x_{n}\right\|=0$. Since $x^{*} \in F(T) \cap \Omega^{*} \cap \Lambda$ and $\alpha_{n}+\sum_{i=1}^{n}\left(\alpha_{i-1}-\right.$ $\left.\alpha_{i}\right)=1$, by using (24) and (25), we obtain

$$
\begin{aligned}
\left\|x_{n+1}-x^{*}\right\|^{2}= & \left\|P_{C}\left[\alpha_{n} f\left(x_{n}\right)+\sum_{i=1}^{n}\left(\alpha_{i-1}-\alpha_{i}\right) V_{i} y_{n}\right]-x^{*}\right\|^{2} \\
\leq & \left\|\alpha_{n} f\left(x_{n}\right)+\sum_{i=1}^{n}\left(\alpha_{i-1}-\alpha_{i}\right) V_{i} y_{n}-x^{*}\right\|^{2} \\
= & \left\|\alpha_{n} f\left(x_{n}\right)+\sum_{i=1}^{n}\left(\alpha_{i-1}-\alpha_{i}\right) V_{i} y_{n}-\alpha_{n} x^{*}-\sum_{i=1}^{n}\left(\alpha_{i-1}-\alpha_{i}\right) V_{i} x^{*}\right\|^{2} \\
\leq & \alpha_{n}\left\|f\left(x_{n}\right)-x^{*}\right\|^{2}+\sum_{i=1}^{n}\left(\alpha_{i-1}-\alpha_{i}\right)\left\|V_{i} y_{n}-V_{i} x^{*}\right\|^{2} \\
\leq & \alpha_{n}\left\|f\left(x_{n}\right)-x^{*}\right\|^{2}+\sum_{i=1}^{n}\left(\alpha_{i-1}-\alpha_{i}\right)\left\|y_{n}-x^{*}\right\|^{2} \\
\leq & \alpha_{n}\left\|f\left(x_{n}\right)-x^{*}\right\|^{2}+\left(1-\alpha_{n}\right)\left(\beta_{n}\left\|S x_{n}-x^{*}\right\|^{2}+\left(1-\beta_{n}\right)\left\|z_{n}-x^{*}\right\|^{2}\right) \\
\leq & \alpha_{n}\left\|f\left(x_{n}\right)-x^{*}\right\|^{2}+\left(1-\alpha_{n}\right) \beta_{n}\left\|S x_{n}-x^{*}\right\|^{2} \\
& +\left(1-\alpha_{n}\right)\left(1-\beta_{n}\right)\left\{\left\|x_{n}-x^{*}\right\|^{2}+\gamma(L \gamma-1)\left\|\left(T_{r_{n}}^{F_{2}}-I\right) A x_{n}\right\|^{2}\right. \\
& \left.-\lambda_{n}\left(2 \alpha-\lambda_{n}\right)\left\|D u_{n}-D x^{*}\right\|^{2}\right\} \\
\leq & \alpha_{n}\left\|f\left(x_{n}\right)-x^{*}\right\|^{2}+\beta_{n}\left\|S x_{n}-x^{*}\right\|^{2}+\left\|x_{n}-x^{*}\right\|^{2} \\
& -\left(1-\alpha_{n}\right)\left(1-\beta_{n}\right)\left\{\gamma(1-L \gamma)\left\|\left(T_{r_{n}}^{F_{2}}-I\right) A x_{n}\right\|^{2}\right. \\
& \left.+\lambda_{n}\left(2 \alpha-\lambda_{n}\right)\left\|D u_{n}-D x^{*}\right\|^{2}\right\} .
\end{aligned}
$$

Then, from the above inequality, we get

$$
\begin{aligned}
& \left(1-\alpha_{n}\right)\left(1-\beta_{n}\right)\left\{\gamma(1-L \gamma)\left\|\left(T_{r_{n}}^{F_{2}}-I\right) A x_{n}\right\|^{2}+\lambda_{n}\left(2 \alpha-\lambda_{n}\right)\left\|D u_{n}-D x^{*}\right\|^{2}\right\} \\
& \quad \leq \alpha_{n}\left\|f\left(x_{n}\right)-x^{*}\right\|^{2}+\beta_{n}\left\|S x_{n}-x^{*}\right\|^{2}+\left\|x_{n}-x^{*}\right\|^{2}-\left\|x_{n+1}-x^{*}\right\|^{2} \\
& \quad \leq \alpha_{n}\left\|f\left(x_{n}\right)-x^{*}\right\|^{2}+\beta_{n}\left\|S x_{n}-x^{*}\right\|^{2}+\left(\left\|x_{n}-x^{*}\right\|+\left\|x_{n+1}-x^{*}\right\|\right)\left\|x_{n+1}-x_{n}\right\| .
\end{aligned}
$$


Since $\gamma(1-L \gamma)>0, \liminf _{n \rightarrow \infty} \lambda_{n} \leq \limsup _{n \rightarrow \infty} \lambda_{n}<2 \alpha, \lim _{n \rightarrow \infty}\left\|x_{n+1}-x_{n}\right\|=0, \alpha_{n} \rightarrow 0$ and $\beta_{n} \rightarrow 0$, we obtain

$$
\lim _{n \rightarrow \infty}\left\|\left(T_{r_{n}}^{F_{2}}-I\right) A x_{n}\right\|=0
$$

and

$$
\lim _{n \rightarrow \infty}\left\|D u_{n}-D x^{*}\right\|=0
$$

Since $T_{r_{n}}^{F_{1}}$ is firmly nonexpansive, we have

$$
\begin{aligned}
\left\|u_{n}-x^{*}\right\|^{2}= & \left\|T_{r_{n}}^{F_{1}}\left(x_{n}+\gamma A^{*}\left(T_{r_{n}}^{F_{2}}-I\right) A x_{n}\right)-T_{r_{n}}^{F_{1}}\left(x^{*}\right)\right\|^{2} \\
\leq & \left\langle u_{n}-x^{*}, x_{n}+\gamma A^{*}\left(T_{r_{n}}^{F_{2}}-I\right) A x_{n}-x^{*}\right\rangle \\
= & \frac{1}{2}\left\{\left\|u_{n}-x^{*}\right\|^{2}+\left\|x_{n}+\gamma A^{*}\left(T_{r_{n}}^{F_{2}}-I\right) A x_{n}-x^{*}\right\|^{2}\right. \\
& \left.-\left\|u_{n}-x^{*}-\left[x_{n}+\gamma A^{*}\left(T_{r_{n}}^{F_{2}}-I\right) A x_{n}-x^{*}\right]\right\|^{2}\right\} \\
= & \frac{1}{2}\left\{\left\|u_{n}-x^{*}\right\|^{2}+\left\|x_{n}+\gamma A^{*}\left(T_{r_{n}}^{F_{2}}-I\right) A x_{n}-x^{*}\right\|^{2}\right. \\
& \left.-\left\|u_{n}-x_{n}-\gamma A^{*}\left(T_{r_{n}}^{F_{2}}-I\right) A x_{n}\right\|^{2}\right\} \\
\leq & \frac{1}{2}\left\{\left\|u_{n}-x^{*}\right\|^{2}+\left\|x_{n}-x^{*}\right\|^{2}-\left\|u_{n}-x_{n}-\gamma A^{*}\left(T_{r_{n}}^{F_{2}}-I\right) A x_{n}\right\|^{2}\right\} \\
= & \frac{1}{2}\left\{\left\|u_{n}-x^{*}\right\|^{2}+\left\|x_{n}-x^{*}\right\|^{2}\right. \\
& \left.-\left[\left\|u_{n}-x_{n}\right\|^{2}+\gamma^{2}\left\|A^{*}\left(T_{r_{n}}^{F_{2}}-I\right) A x_{n}\right\|^{2}-2 \gamma\left\langle u_{n}-x_{n}, A^{*}\left(T_{r_{n}}^{F_{2}}-I\right) A x_{n}\right\rangle\right]\right\},
\end{aligned}
$$

where the last inequality follows from (21) and (24). Hence, we get

$$
\left\|u_{n}-x^{*}\right\|^{2} \leq\left\|x_{n}-x^{*}\right\|^{2}-\left\|u_{n}-x_{n}\right\|^{2}+2 \gamma\left\|A u_{n}-A x_{n}\right\|\left\|\left(T_{r_{n}}^{F_{2}}-I\right) A x_{n}\right\| .
$$

From (34), (25) and the above inequality, we have

$$
\begin{aligned}
\left\|x_{n+1}-x^{*}\right\|^{2} \leq & \alpha_{n}\left\|f\left(x_{n}\right)-x^{*}\right\|^{2}+\left(1-\alpha_{n}\right)\left(\beta_{n}\left\|S x_{n}-x^{*}\right\|^{2}+\left(1-\beta_{n}\right)\left\|z_{n}-x^{*}\right\|^{2}\right) \\
\leq & \alpha_{n}\left\|f\left(x_{n}\right)-x^{*}\right\|^{2}+\left(1-\alpha_{n}\right)\left(\beta_{n}\left\|S x_{n}-x^{*}\right\|^{2}+\left(1-\beta_{n}\right)\left\|u_{n}-x^{*}\right\|^{2}\right) \\
\leq & \alpha_{n}\left\|f\left(x_{n}\right)-x^{*}\right\|^{2}+\left(1-\alpha_{n}\right)\left\{\beta_{n}\left\|S x_{n}-x^{*}\right\|^{2}\right. \\
& \left.+\left(1-\beta_{n}\right)\left(\left\|x_{n}-x^{*}\right\|^{2}-\left\|u_{n}-x_{n}\right\|^{2}+2 \gamma\left\|A u_{n}-A x_{n}\right\|\left\|\left(T_{r_{n}}^{F_{2}}-I\right) A x_{n}\right\|\right)\right\} \\
\leq & \alpha_{n}\left\|f\left(x_{n}\right)-x^{*}\right\|^{2}+\beta_{n}\left\|S x_{n}-x^{*}\right\|^{2}+\left\|x_{n}-x^{*}\right\|^{2} \\
& \quad\left(1-\alpha_{n}\right)\left(1-\beta_{n}\right)\left\|u_{n}-x_{n}\right\|^{2}+2 \gamma\left\|A u_{n}-A x_{n}\right\|\left\|\left(T_{r_{n}}^{F_{2}}-I\right) A x_{n}\right\| .
\end{aligned}
$$

Hence

$$
\begin{aligned}
& \left(1-\alpha_{n}\right)\left(1-\beta_{n}\right)\left\|u_{n}-x_{n}\right\|^{2} \\
& \quad \leq \alpha_{n}\left\|f\left(x_{n}\right)-x^{*}\right\|^{2}+\beta_{n}\left\|S x_{n}-x^{*}\right\|^{2}+\left\|x_{n}-x^{*}\right\|^{2}-\left\|x_{n+1}-x^{*}\right\|^{2}
\end{aligned}
$$




$$
\begin{aligned}
& +2 \gamma\left\|A u_{n}-A x_{n}\right\|\left\|\left(T_{r_{n}}^{F_{2}}-I\right) A x_{n}\right\| \\
\leq & \alpha_{n}\left\|f\left(x_{n}\right)-x^{*}\right\|^{2}+\beta_{n}\left\|S x_{n}-x^{*}\right\|^{2}+\left(\left\|x_{n}-x^{*}\right\|+\left\|x_{n+1}-x^{*}\right\|\right)\left\|x_{n+1}-x_{n}\right\| \\
& +2 \gamma\left\|A u_{n}-A x_{n}\right\|\left\|\left(T_{r_{n}}^{F_{2}}-I\right) A x_{n}\right\| .
\end{aligned}
$$

Since $\lim _{n \rightarrow \infty}\left\|x_{n+1}-x_{n}\right\|=0, \alpha_{n} \rightarrow 0, \beta_{n} \rightarrow 0$ and $\lim _{n \rightarrow \infty}\left\|\left(T_{r_{n}}^{F_{2}}-I\right) A x_{n}\right\|=0$, we obtain

$$
\lim _{n \rightarrow \infty}\left\|u_{n}-x_{n}\right\|=0
$$

From (17), we get

$$
\begin{aligned}
\left\|z_{n}-x^{*}\right\|^{2}= & \left\|P_{C}\left[u_{n}-\lambda_{n} D u_{n}\right]-P_{C}\left[x^{*}-\lambda_{n} D x^{*}\right]\right\|^{2} \\
\leq & \left\langle z_{n}-x^{*},\left(u_{n}-\lambda_{n} D u_{n}\right)-\left(x^{*}-\lambda_{n} D x^{*}\right)\right\rangle \\
= & \frac{1}{2}\left\{\left\|z_{n}-x^{*}\right\|^{2}+\left\|u_{n}-x^{*}-\lambda_{n}\left(D u_{n}-D x^{*}\right)\right\|^{2}\right. \\
& \left.-\left\|u_{n}-x^{*}-\lambda_{n}\left(D u_{n}-D x^{*}\right)-\left(z_{n}-x^{*}\right)\right\|^{2}\right\} \\
\leq & \frac{1}{2}\left\{\left\|z_{n}-x^{*}\right\|^{2}+\left\|u_{n}-x^{*}\right\|^{2}-\left\|u_{n}-z_{n}-\lambda_{n}\left(D u_{n}-D x^{*}\right)\right\|^{2}\right\} \\
\leq & \frac{1}{2}\left\{\left\|z_{n}-x^{*}\right\|^{2}+\left\|u_{n}-x^{*}\right\|^{2}-\left\|u_{n}-z_{n}\right\|^{2}+2 \lambda_{n}\left\langle u_{n}-z_{n}, D u_{n}-D x^{*}\right\rangle\right\} \\
\leq & \frac{1}{2}\left\{\left\|z_{n}-x^{*}\right\|^{2}+\left\|u_{n}-x^{*}\right\|^{2}-\left\|u_{n}-z_{n}\right\|^{2}+2 \lambda_{n}\left\|u_{n}-z_{n}\right\|\left\|D u_{n}-D x^{*}\right\|\right\} .
\end{aligned}
$$

Hence

$$
\begin{aligned}
\left\|z_{n}-x^{*}\right\|^{2} & \leq\left\|u_{n}-x^{*}\right\|^{2}-\left\|u_{n}-z_{n}\right\|^{2}+2 \lambda_{n}\left\|u_{n}-z_{n}\right\|\left\|D u_{n}-D x^{*}\right\| \\
& \leq\left\|x_{n}-x^{*}\right\|^{2}-\left\|u_{n}-z_{n}\right\|^{2}+2 \lambda_{n}\left\|u_{n}-z_{n}\right\|\left\|D u_{n}-D x^{*}\right\| .
\end{aligned}
$$

From (34) and the above inequality, we have

$$
\begin{aligned}
\left\|x_{n+1}-x^{*}\right\|^{2} \leq & \alpha_{n}\left\|f\left(x_{n}\right)-x^{*}\right\|^{2}+\left(1-\alpha_{n}\right)\left(\beta_{n}\left\|S x_{n}-x^{*}\right\|^{2}+\left(1-\beta_{n}\right)\left\|z_{n}-x^{*}\right\|^{2}\right) \\
\leq & \alpha_{n}\left\|f\left(x_{n}\right)-x^{*}\right\|^{2}+\left(1-\alpha_{n}\right)\left\{\beta_{n}\left\|S x_{n}-x^{*}\right\|^{2}\right. \\
& \left.+\left(1-\beta_{n}\right)\left(\left\|x_{n}-x^{*}\right\|^{2}-\left\|u_{n}-z_{n}\right\|^{2}+2 \lambda_{n}\left\|u_{n}-z_{n}\right\|\left\|D u_{n}-D x^{*}\right\|\right)\right\} \\
\leq & \alpha_{n}\left\|f\left(x_{n}\right)-x^{*}\right\|^{2}+\beta_{n}\left\|S x_{n}-x^{*}\right\|^{2}+\left\|x_{n}-x^{*}\right\|^{2} \\
& -\left(1-\alpha_{n}\right)\left(1-\beta_{n}\right)\left\|u_{n}-z_{n}\right\|^{2}+2 \lambda_{n}\left\|u_{n}-z_{n}\right\|\left\|D u_{n}-D x^{*}\right\| .
\end{aligned}
$$

Hence

$$
\begin{aligned}
&\left(1-\alpha_{n}\right)\left(1-\beta_{n}\right)\left\|u_{n}-z_{n}\right\|^{2} \\
& \leq \alpha_{n}\left\|f\left(x_{n}\right)-x^{*}\right\|^{2}+\beta_{n}\left\|S x_{n}-x^{*}\right\|^{2}+\left\|x_{n}-x^{*}\right\|^{2}-\left\|x_{n+1}-x^{*}\right\|^{2} \\
&+2 \lambda_{n}\left\|u_{n}-z_{n}\right\|\left\|D u_{n}-D x^{*}\right\| \\
& \leq \alpha_{n}\left\|f\left(x_{n}\right)-x^{*}\right\|^{2}+\beta_{n}\left\|S x_{n}-x^{*}\right\|^{2}+\left(\left\|x_{n}-x^{*}\right\|+\left\|x_{n+1}-x^{*}\right\|\right)\left\|x_{n+1}-x_{n}\right\| \\
&+2 \lambda_{n}\left\|u_{n}-z_{n}\right\|\left\|D u_{n}-D x^{*}\right\| .
\end{aligned}
$$


Since $\lim _{n \rightarrow \infty}\left\|x_{n+1}-x_{n}\right\|=0, \alpha_{n} \rightarrow 0, \beta_{n} \rightarrow 0$ and $\lim _{n \rightarrow \infty}\left\|D u_{n}-D x^{*}\right\|=0$, we obtain

$$
\lim _{n \rightarrow \infty}\left\|u_{n}-z_{n}\right\|=0
$$

It follows from (36) and (37) that

$$
\lim _{n \rightarrow \infty}\left\|x_{n}-z_{n}\right\|=0
$$

Now, let $z \in F(T) \cap \Omega^{*} \cap \Lambda$, since for each $i \geq 1, V_{i} x_{n} \in C$ and $\alpha_{n}+\sum_{i=1}^{n}\left(\alpha_{i-1}-\alpha_{i}\right)=1$, we have $\sum_{i=1}^{n}\left(\alpha_{i-1}-\alpha_{i}\right) V_{i} x_{n}+\alpha_{n} z \in C$, and

$$
\begin{aligned}
\sum_{i=1}^{n}\left(\alpha_{i-1}-\alpha_{i}\right)\left(x_{n}-V_{i} x_{n}\right) & \\
=P_{C} & {\left[\alpha_{n} f\left(x_{n}\right)+\sum_{i=1}^{n}\left(\alpha_{i-1}-\alpha_{i}\right) V_{i} y_{n}\right]+\left(1-\alpha_{n}\right) x_{n} } \\
& -\left(\sum_{i=1}^{n}\left(\alpha_{i-1}-\alpha_{i}\right) V_{i} x_{n}+\alpha_{n} z\right)+\alpha_{n} z-x_{n+1} \\
= & P_{C}\left[\alpha_{n} f\left(x_{n}\right)+\sum_{i=1}^{n}\left(\alpha_{i-1}-\alpha_{i}\right) V_{i} y_{n}\right]+\alpha_{n}\left(z-x_{n+1}\right) \\
& -P_{C}\left[\sum_{i=1}^{n}\left(\alpha_{i-1}-\alpha_{i}\right) V_{i} x_{n}+\alpha_{n} z\right]+\left(1-\alpha_{n}\right)\left(x_{n}-x_{n+1}\right) .
\end{aligned}
$$

It follows that

$$
\begin{aligned}
\sum_{i=1}^{n}( & \left.\alpha_{i-1}-\alpha_{i}\right)\left\langle x_{n}-V_{i} x_{n}, x_{n}-x^{*}\right\rangle \\
= & \left\langle P_{C}\left[\alpha_{n} f\left(x_{n}\right)+\sum_{i=1}^{n}\left(\alpha_{i-1}-\alpha_{i}\right) V_{i} y_{n}\right]-P_{C}\left[\sum_{i=1}^{n}\left(\alpha_{i-1}-\alpha_{i}\right) V_{i} x_{n}+\alpha_{n} z\right], x_{n}-x^{*}\right\rangle \\
& +\alpha_{n}\left\langle z-x_{n+1}, x_{n}-x^{*}\right\rangle+\left(1-\alpha_{n}\right)\left\langle x_{n}-x_{n+1}, x_{n}-x^{*}\right\rangle \\
\leq & \left\|\alpha_{n}\left(f\left(x_{n}\right)-z\right)+\sum_{i=1}^{n}\left(\alpha_{i-1}-\alpha_{i}\right)\left(V_{i} y_{n}-V_{i} x_{n}\right)\right\|\left\|x_{n}-x^{*}\right\| \\
& +\alpha_{n}\left\|z-x_{n+1}\right\|\left\|x_{n}-x^{*}\right\|+\left(1-\alpha_{n}\right)\left\|x_{n}-x_{n+1}\right\|\left\|x_{n}-x^{*}\right\| \\
\leq & \alpha_{n}\left\|f\left(x_{n}\right)-z\right\|\left\|x_{n}-x^{*}\right\|+\sum_{i=1}^{n}\left(\alpha_{i-1}-\alpha_{i}\right)\left\|y_{n}-x_{n}\right\|\left\|x_{n}-x^{*}\right\| \\
& +\alpha_{n}\left\|z-x_{n+1}\right\|\left\|x_{n}-x^{*}\right\|+\left(1-\alpha_{n}\right)\left\|x_{n}-x_{n+1}\right\|\left\|x_{n}-x^{*}\right\| \\
= & \alpha_{n}\left\|f\left(x_{n}\right)-z\right\|\left\|x_{n}-x^{*}\right\|+\left(1-\alpha_{n}\right)\left\|y_{n}-x_{n}\right\|\left\|x_{n}-x^{*}\right\| \\
& +\alpha_{n}\left\|z-x_{n+1}\right\|\left\|x_{n}-x^{*}\right\|+\left(1-\alpha_{n}\right)\left\|x_{n}-x_{n+1}\right\|\left\|x_{n}-x^{*}\right\| \\
\leq & \alpha_{n}\left\|f\left(x_{n}\right)-z\right\|\left\|x_{n}-x^{*}\right\| \\
& +\left(1-\alpha_{n}\right)\left\|\beta_{n} S x_{n}+\left(1-\beta_{n}\right) z_{n}-x_{n}\right\|\left\|x_{n}-x^{*}\right\|+\alpha_{n}\left\|z-x_{n+1}\right\|\left\|x_{n}-x^{*}\right\|
\end{aligned}
$$




$$
\begin{aligned}
& +\left(1-\alpha_{n}\right)\left\|x_{n}-x_{n+1}\right\|\left\|x_{n}-x^{*}\right\| \\
\leq & \alpha_{n}\left\|f\left(x_{n}\right)-z\right\|\left\|x_{n}-x^{*}\right\|+\left(1-\alpha_{n}\right) \beta_{n}\left\|S x_{n}-x_{n}\right\|\left\|x_{n}-x^{*}\right\| \\
& +\left(1-\alpha_{n}\right)\left(1-\beta_{n}\right)\left\|z_{n}-x_{n}\right\|\left\|x_{n}-x^{*}\right\| \\
& +\alpha_{n}\left\|z-x_{n+1}\right\|\left\|x_{n}-x^{*}\right\|+\left(1-\alpha_{n}\right)\left\|x_{n}-x_{n+1}\right\|\left\|x_{n}-x^{*}\right\| .
\end{aligned}
$$

From Lemma 2.9 and the above inequality, we get

$$
\begin{aligned}
& \frac{1}{2} \sum_{i=1}^{n}\left(\alpha_{i-1}-\alpha_{i}\right)\left\|x_{n}-V_{i} x_{n}\right\|^{2} \\
& \leq \sum_{i=1}^{n}\left(\alpha_{i-1}-\alpha_{i}\right)\left(x_{n}-V_{i} x_{n}, x_{n}-x^{*}\right) \\
& \leq \alpha_{n}\left\|f\left(x_{n}\right)-z\right\|\left\|x_{n}-x^{*}\right\|+\left(1-\alpha_{n}\right) \beta_{n}\left\|S x_{n}-x_{n}\right\|\left\|x_{n}-x^{*}\right\| \\
& \quad+\left(1-\alpha_{n}\right)\left(1-\beta_{n}\right)\left\|z_{n}-x_{n}\right\|\left\|x_{n}-x^{*}\right\|+\alpha_{n}\left\|z-x_{n+1}\right\|\left\|x_{n}-x^{*}\right\| \\
& \quad+\left(1-\alpha_{n}\right)\left\|x_{n}-x_{n+1}\right\|\left\|x_{n}-x^{*}\right\| .
\end{aligned}
$$

Since $\lim _{n \rightarrow \infty}\left\|x_{n+1}-x_{n}\right\|=0, \alpha_{n} \rightarrow 0, \beta_{n} \rightarrow 0$ and $\lim _{n \rightarrow \infty}\left\|x_{n}-z_{n}\right\|=0$, we obtain

$$
\lim _{n \rightarrow \infty} \sum_{i=1}^{n}\left(\alpha_{i-1}-\alpha_{i}\right)\left\|x_{n}-V_{i} x_{n}\right\|^{2}=0
$$

Since $\left(\alpha_{i-1}-\alpha_{i}\right)\left\|x_{n}-V_{i} x_{n}\right\|^{2} \leq \sum_{i=1}^{n}\left(\alpha_{i-1}-\alpha_{i}\right)\left\|x_{n}-V_{i} x_{n}\right\|^{2}$ and $\left\{\alpha_{n}\right\}$ is strictly decreasing, we have

$$
\lim _{n \rightarrow \infty}\left\|x_{n}-V_{i} x_{n}\right\|=0
$$

Hence, we obtain

$$
\lim _{n \rightarrow \infty}\left\|x_{n}-T_{i} x_{n}\right\|=\lim _{n \rightarrow \infty} \frac{\left\|x_{n}-V_{i} x_{n}\right\|}{\left(1-k_{i}\right)}=0, \quad \forall i \geq 1 .
$$

Since $\left\{x_{n}\right\}$ is bounded, without loss of generality, we can assume that $x_{n} \rightarrow w \in C$. It follows from Lemma 2.4 that $w \in F(T)$. Therefore $w_{w}\left(x_{n}\right) \subset F(T)$.

Theorem 3.1 The sequence $\left\{x_{n}\right\}$ generated by Algorithm 3.1 converges strongly to $z=$ $P_{\Omega^{*} \cap \Lambda \cap F(T)} f(z)$, which is the unique solution of the variational inequality

$$
\langle(I-f) z, x-z\rangle \geq 0, \quad \forall x \in \Omega^{*} \cap \Lambda \cap F(T),
$$

which is the optimality condition for a minimization problem

$$
\min _{x \in \Upsilon}\left\{\frac{1}{2}\|x\|^{2}-h(x)\right\}
$$

where $h$ is a potential function for $f\left(\right.$ i.e., $h^{\prime}(x)=f(x)$ for $x \in H$ ) and $\Upsilon=\Omega^{*} \cap \Lambda \cap F(T)$. 
Proof Since $\left\{x_{n}\right\}$ is bounded $x_{n} \rightarrow w$ and from Lemma 3.2, we have $w \in F(T)$. Next, we show that $w \in E P\left(F_{1}\right)$. Since $u_{n}=T_{r_{n}}^{F_{1}}\left(x_{n}+\gamma A^{*}\left(T_{r_{n}}^{F_{2}}-I\right) A x_{n}\right)$, we have

$$
F_{1}\left(u_{n}, y\right)+\frac{1}{r_{n}}\left\langle y-u_{n}, u_{n}-x_{n}\right\rangle-\frac{1}{r_{n}}\left\langle y-u_{n}, \gamma A^{*}\left(T_{r_{n}}^{F_{2}}-I\right) A x_{n}\right\rangle \geq 0, \quad \forall y \in C .
$$

It follows from the monotonicity of $F_{1}$ that

$$
-\frac{1}{r_{n}}\left\langle y-u_{n}, \gamma A^{*}\left(T_{r_{n}}^{F_{2}}-I\right) A x_{n}\right\rangle+\frac{1}{r_{n}}\left\langle y-u_{n}, u_{n}-x_{n}\right\rangle \geq F_{1}\left(y, u_{n}\right), \quad \forall y \in C
$$

and

$$
-\frac{1}{r_{n_{k}}}\left\langle y-u_{n_{k}}, \gamma A^{*}\left(T_{r_{n_{k}}}^{F_{2}}-I\right) A x_{n_{k}}\right\rangle+\left\langle y-u_{n_{k}}, \frac{u_{n_{k}}-x_{n_{k}}}{r_{n_{k}}}\right\rangle \geq F_{1}\left(y, u_{n_{k}}\right), \quad \forall y \in C .
$$

Since $\lim _{n \rightarrow \infty}\left\|u_{n}-x_{n}\right\|=0, \lim _{n \rightarrow \infty}\left\|\left(T_{r_{n}}^{F_{2}}-I\right) A x_{n}\right\|=0$ and $x_{n} \rightarrow w$, it is easy to observe that $u_{n_{k}} \rightarrow w$. It follows by Assumption 2.1(iv) that $F_{1}(y, w) \leq 0, \forall y \in C$.

For any $0<t \leq 1$ and $y \in C$, let $y_{t}=t y+(1-t) w$, we have $y_{t} \in C$. Then, from Assumption 2.1(i) and (iv), we have

$$
\begin{aligned}
0 & =F_{1}\left(y_{t}, y_{t}\right) \leq t F_{1}\left(y_{t}, y\right)+(1-t) F_{1}\left(y_{t}, w\right) \\
& \leq t F_{1}\left(y_{t}, y\right) .
\end{aligned}
$$

Therefore $F_{1}\left(y_{t}, y\right) \geq 0$. From Assumption 2.1(iii), we have $F_{1}(w, y) \geq 0$, which implies that $w \in E P\left(F_{1}\right)$.

Next, we show that $A w \in E P\left(F_{2}\right)$. Since $\left\{x_{n}\right\}$ is bounded and $x_{n} \rightarrow w$, there exists a subsequence $\left\{x_{n_{k}}\right\}$ of $\left\{x_{n}\right\}$ such that $x_{n_{k}} \rightarrow w$ and since $A$ is a bounded linear operator so that $A x_{n_{k}} \rightarrow A w$. Now set $v_{n_{k}}=A x_{n_{k}}-T_{r_{n_{k}}}^{F_{2}} A x_{n_{k}}$. It follows from (35) that $\lim _{k \rightarrow \infty} v_{n_{k}}=0$ and $A x_{n_{k}}-v_{n_{k}}=T_{r_{n_{k}}}^{F_{2}} A x_{n_{k}}$. Therefore from the definition of $T_{r_{n_{k}}}^{F_{2}}$, we have

$$
F_{2}\left(A x_{n_{k}}-v_{n_{k}}, y\right)+\frac{1}{r_{n_{k}}}\left\langle y-\left(A x_{n_{k}}-v_{n_{k}}\right),\left(A x_{n_{k}}-v_{n_{k}}\right)-A x_{n_{k}}\right\rangle \geq 0, \quad \forall y \in C .
$$

Since $F_{2}$ is upper semicontinuous in the first argument, taking limsup to the above inequality as $k \rightarrow \infty$ and using Assumption 2.1(iv), we obtain

$$
F_{2}(A w, y) \geq 0, \quad \forall y \in C,
$$

which implies that $A w \in E P\left(F_{2}\right)$ and hence $w \in \Lambda$.

Furthermore, we show that $w \in \Omega^{*}$. Let

$$
T v= \begin{cases}D v+N_{C} v, & \forall v \in C, \\ \emptyset, & \text { otherwise }\end{cases}
$$

where $N_{C} v:=\{w \in H:\langle w, v-u\rangle \geq 0, \forall u \in C\}$ is the normal cone to $C$ at $v \in C$. Then $T$ is maximal monotone and $0 \in T v$ if and only if $v \in \Omega^{*}$ (see [25]). Let $G(T)$ denote the graph 
of $T$ and let $(v, u) \in G(T)$. Since $u-D v \in N_{C} v$ and $z_{n} \in C$, we have

$$
\left\langle v-z_{n}, u-D v\right\rangle \geq 0 .
$$

On the other hand, it follows from $z_{n}=P_{C}\left[u_{n}-\lambda_{n} D u_{n}\right]$ and $v \in C$ that

$$
\left\langle v-z_{n}, z_{n}-\left(u_{n}-\lambda_{n} D u_{n}\right)\right\rangle \geq 0
$$

and

$$
\left\langle v-z_{n}, \frac{z_{n}-u_{n}}{\lambda_{n}}+D u_{n}\right\rangle \geq 0
$$

Therefore, from (41) and inverse strong monotonicity of $D$, we have

$$
\begin{aligned}
\left\langle v-z_{n_{k}}, u\right\rangle & \geq\left\langle v-z_{n_{k}}, D v\right\rangle \\
& \geq\left\langle v-z_{n_{k}}, D v\right\rangle-\left\langle v-z_{n_{k}}, \frac{z_{n_{k}}-u_{n_{k}}}{\lambda_{n_{k}}}+D u_{n_{k}}\right\rangle \\
& \geq\left\langle v-z_{n_{k}}, D v-D z_{n_{k}}\right\rangle+\left\langle v-z_{n_{k}}, D z_{n_{k}}-D u_{n_{k}}\right\rangle-\left\langle v-z_{n_{k}}, \frac{z_{n_{k}}-u_{n_{k}}}{\lambda_{n_{k}}}\right\rangle \\
& \geq\left\langle v-z_{n_{k}}, D z_{n_{k}}-D u_{n_{k}}\right\rangle-\left\langle v-z_{n_{k}}, \frac{z_{n_{k}}-u_{n_{k}}}{\lambda_{n_{k}}}\right\rangle .
\end{aligned}
$$

Since $\lim _{n \rightarrow \infty}\left\|u_{n}-z_{n}\right\|=0$ and $u_{n_{k}} \rightarrow w$, it is easy to observe that $z_{n_{k}} \rightarrow w$. Hence, we obtain $\langle v-w, u\rangle \geq 0$. Since $T$ is maximal monotone, we have $w \in T^{-1} 0$ and hence $w \in \Omega^{*}$. Thus we have

$$
w \in \Omega^{*} \cap \Lambda \cap F(T) .
$$

Since $\Omega^{*}, \Lambda$ and $F(T)$ are convex, then $\Omega^{*} \cap \Lambda \cap F(T)$ is convex. Next, we claim that $\limsup _{n \rightarrow \infty}\left\langle f(z)-z, x_{n}-z\right\rangle \leq 0$, where $z=P_{\Omega^{*} \cap \Lambda \cap F(T)} f(z)$.

Since $\left\{x_{n}\right\}$ is bounded, there exists a subsequence $\left\{x_{n_{k}}\right\}$ of $\left\{x_{n}\right\}$ such that

$$
\limsup _{n \rightarrow \infty}\left\langle f(z)-z, x_{n}-z\right\rangle=\limsup _{k \rightarrow \infty}\left\langle f(z)-z, x_{n_{k}}-z\right\rangle=\langle f(z)-z, w-z\rangle \leq 0 .
$$

Next, we show that $x_{n} \rightarrow z$. From (16), we get

$$
\begin{aligned}
\left\|x_{n+1}-z\right\|^{2}= & \left\langle x_{n+1}-\alpha_{n} f\left(x_{n}\right)-\sum_{i=1}^{n}\left(\alpha_{i-1}-\alpha_{i}\right) V_{i} y_{n}, x_{n+1}-z\right\rangle \\
& +\left\langle\alpha_{n} f\left(x_{n}\right)+\sum_{i=1}^{n}\left(\alpha_{i-1}-\alpha_{i}\right) V_{i} y_{n}-z, x_{n+1}-z\right\rangle \\
\leq & \left\langle\alpha_{n} f\left(x_{n}\right)+\sum_{i=1}^{n}\left(\alpha_{i-1}-\alpha_{i}\right) V_{i} y_{n}-z, x_{n+1}-z\right\rangle \\
\leq & \alpha_{n}\left\langle f\left(x_{n}\right)-f(z), x_{n+1}-z\right\rangle+\alpha_{n}\left\langle f(z)-z, x_{n+1}-z\right\rangle
\end{aligned}
$$




$$
\begin{aligned}
& +\sum_{i=1}^{n}\left(\alpha_{i-1}-\alpha_{i}\right)\left\langle V_{i} y_{n}-z, x_{n+1}-z\right\rangle \\
\leq & \alpha_{n}\left\|f\left(x_{n}\right)-f(z)\right\|\left\|x_{n+1}-z\right\|+\alpha_{n}\left\langle f(z)-z, x_{n+1}-z\right\rangle \\
& +\sum_{i=1}^{n}\left(\alpha_{i-1}-\alpha_{i}\right)\left\|V_{i} y_{n}-z\right\|\left\|x_{n+1}-z\right\| \\
\leq & \alpha_{n} \rho\left\|x_{n}-z\right\|\left\|x_{n+1}-z\right\|+\alpha_{n}\left\langle f(z)-z, x_{n+1}-z\right\rangle \\
& +\sum_{i=1}^{n}\left(\alpha_{i-1}-\alpha_{i}\right)\left\|y_{n}-z\right\|\left\|x_{n+1}-z\right\| \\
\leq & \alpha_{n} \rho\left\|x_{n}-z\right\|\left\|x_{n+1}-z\right\|+\alpha_{n}\left\langle f(z)-z, x_{n+1}-z\right\rangle \\
& +\left(1-\alpha_{n}\right)\left\{\beta_{n}\left\|S x_{n}-S z\right\|+\beta_{n}\|S z-z\|+\left(1-\beta_{n}\right)\left\|z_{n}-z\right\|\right\}\left\|x_{n+1}-z\right\| \\
\leq & \alpha_{n} \rho\left\|x_{n}-z\right\|\left\|x_{n+1}-z\right\|+\alpha_{n}\left\langle f(z)-z, x_{n+1}-z\right\rangle \\
& +\left(1-\alpha_{n}\right)\left\{\beta_{n}\left\|x_{n}-z\right\|+\beta_{n}\|S z-z\|+\left(1-\beta_{n}\right)\left\|x_{n}-z\right\|\right\}\left\|x_{n+1}-z\right\| \\
\leq & \left(1-\alpha_{n}(1-\rho)\right)\left\|x_{n}-z\right\|\left\|x_{n+1}-z\right\|+\alpha_{n}\left\langle f(z)-z, x_{n+1}-z\right\rangle \\
& +\left(1-\alpha_{n}\right) \beta_{n}\|S z-z\|\left\|x_{n+1}-z\right\| \\
\leq & \frac{1-\alpha_{n}(1-\rho)}{2}\left(\left\|x_{n}-z\right\|^{2}+\left\|x_{n+1}-z\right\|^{2}\right)+\alpha_{n}\left\langle f(z)-z, x_{n+1}-z\right\rangle \\
& +\left(1-\alpha_{n}\right) \beta_{n}\|S z-z\|\left\|x_{n+1}-z\right\|, \\
& +(1-z)
\end{aligned}
$$

which implies that

$$
\begin{aligned}
\left\|x_{n+1}-z\right\|^{2} \leq & \left(1-\frac{2 \alpha_{n}(1-\rho)}{1+\alpha_{n}(1-\rho)}\right)\left\|x_{n}-z\right\|^{2}+\frac{2 \alpha_{n}}{1+\alpha_{n}(1-\rho)}\left\langle f(z)-z, x_{n+1}-z\right\rangle \\
& +\frac{2\left(1-\alpha_{n}\right) \beta_{n}}{1+\alpha_{n}(1-\rho)}\|S z-z\|\left\|x_{n+1}-z\right\| \\
\leq & \left(1-\frac{2 \alpha_{n}(1-\rho)}{1+\alpha_{n}(1-\rho)}\right)\left\|x_{n}-z\right\|^{2}+\frac{2 \alpha_{n}(1-\rho)}{1+\alpha_{n}(1-\rho)}\left\{\frac{1}{1-\rho}\left\langle f(z)-z, x_{n+1}-z\right\rangle\right. \\
& \left.+\frac{\left(1-\alpha_{n}\right) \beta_{n}}{\alpha_{n}(1-\rho)}\|S z-z\|\left\|x_{n+1}-z\right\|\right\} .
\end{aligned}
$$

Let $\gamma_{n}=\frac{2 \alpha_{n}(1-\rho)}{1+\alpha_{n}(1-\rho)}$ and $\delta_{n}=\frac{2 \alpha_{n}(1-\rho)}{1+\alpha_{n}(1-\rho)}\left\{\frac{1}{1-\rho}\left\langle f(z)-z, x_{n+1}-z\right\rangle+\frac{\left(1-\alpha_{n}\right) \beta_{n}}{\alpha_{n}(1-\rho)}\|S z-z\|\left\|x_{n+1}-z\right\|\right\}$.

Since

$$
\begin{aligned}
& \sum_{n=1}^{\infty} \alpha_{n}=\infty, \quad 1+\alpha_{n}(1-\rho) \leq 2 \text { and } \\
& \limsup _{n \rightarrow \infty}\left\{\frac{1}{1-\rho}\left\langle f(z)-z, x_{n+1}-z\right\rangle+\frac{\left(1-\alpha_{n}\right) \beta_{n}}{\alpha_{n}(1-\rho)}\|S z-z\|\left\|x_{n+1}-z\right\|\right\} \leq 0,
\end{aligned}
$$

it follows that

$$
\sum_{n=1}^{\infty} \gamma_{n}=\infty \quad \text { and } \quad \limsup _{n \rightarrow \infty} \frac{\delta_{n}}{\gamma_{n}} \leq 0
$$

Thus all the conditions of Lemma 2.6 are satisfied. Hence we deduce that $x_{n} \rightarrow z$. 
$P_{\Omega^{*} \cap \Lambda \cap F(T)} f$ is a contraction, there exists a unique $z \in C$ such that $z=P_{\Omega^{*} \cap \Lambda \cap F(T)} f(z)$. From (16), it follows that $z$ is the unique solution of problem (39). This completes the proof.

Theorem 3.2 Let $H_{1}$ and $H_{2}$ be two real Hilbert spaces and $C \subseteq H_{1}$ and $Q \subseteq H_{2}$ be nonempty closed convex subsets of Hilbert spaces $H_{1}$ and $H_{2}$, respectively. Let $A: H_{1} \rightarrow H_{2}$ be a bounded linear operator. Let $D: C \rightarrow H_{1}$ be an $\alpha$-inverse strongly monotone mapping. Assume that $F_{1}: C \times C \rightarrow \mathbb{R}$ and $F_{2}: Q \times Q \rightarrow \mathbb{R}$ are the bifunctions satisfying Assumption 2.1 and $F_{2}$ is upper semicontinuous in the first argument. Let $S: C \rightarrow H_{1}$ be a nonexpansive mapping and $\left\{T_{i}\right\}_{i=1}^{\infty}: C \rightarrow C$ be a countable family of $k_{i}$-strict pseudo-contraction mappings such that $F(T) \cap \Omega^{*} \cap \Lambda \neq \emptyset$, where $F(T)=\bigcap_{i=1}^{\infty} F\left(T_{i}\right)$. Let $f$ be a $\rho$-contraction mapping. For a given $x_{0} \in C$ arbitrarily, let the iterative sequences $\left\{u_{n}\right\},\left\{x_{n}\right\},\left\{y_{n}\right\}$ and $\left\{z_{n}\right\}$ be generated by

$$
\begin{aligned}
& u_{n}=T_{r_{n}}^{F_{1}}\left(x_{n}+\gamma A^{*}\left(T_{r_{n}}^{F_{2}}-I\right) A x_{n}\right) ; \\
& z_{n}=P_{C}\left[u_{n}-\lambda_{n} A u_{n}\right] ; \\
& y_{n}=\beta_{n} S x_{n}+\left(1-\beta_{n}\right) z_{n} ; \\
& x_{n+1}=P_{C}\left[\alpha_{n} f\left(x_{n}\right)+\sum_{i=1}^{n}\left(\alpha_{i-1}-\alpha_{i}\right) V_{i} y_{n}\right], \quad \forall n \geq 0,
\end{aligned}
$$

where $V_{i}=k_{i} I+\left(1-k_{i}\right) T_{i}, 0 \leq k_{i}<1,\left\{r_{n}\right\} \subset(0, \infty),\left\{\lambda_{n}\right\} \subset(0,2 \alpha)$ and $\gamma \in(0,1 / L), L$ is the spectral radius of the operator $A^{*} A$ and $A^{*}$ is the adjoint of $A$ and $\alpha_{0}=1,\left\{\alpha_{n}\right\}$ is a strictly decreasing sequence in $(0,1)$ and $\left\{\beta_{n}\right\}$ is a sequence in $(0,1)$ satisfying the following conditions:

(a) $\lim _{n \rightarrow \infty} \alpha_{n}=0$ and $\sum_{n=1}^{\infty} \alpha_{n}=\infty$,

(b) $\lim _{n \rightarrow \infty} \frac{\beta_{n}}{\alpha_{n}}=\tau \in(0, \infty)$,

(c) $\sum_{n=1}^{\infty}\left(\alpha_{n-1}-\alpha_{n}\right)<\infty$ and $\sum_{n=1}^{\infty}\left|\beta_{n-1}-\beta_{n}\right|<\infty$,

(d) $\lim _{n \rightarrow \infty} \frac{\frac{1}{\mu}\left|r_{n}-r_{n-1}\right|+\left|\lambda_{n}-\lambda_{n-1}\right|+\left|\alpha_{n-1}-\alpha_{n}\right|+\left|\beta_{n-1}-\beta_{n}\right|}{\alpha_{n} \beta_{n}}=0$,

(e) there exists a constant $K>0$ such that $\frac{1}{\alpha_{n}}\left|\frac{1}{\beta_{n}}-\frac{1}{\beta_{n-1}}\right| \leq K$,

(f) $\liminf _{n \rightarrow \infty} r_{n}>0$ and $\sum_{n=1}^{\infty}\left|r_{n-1}-r_{n}\right|<\infty$,

(g) $\liminf _{n \rightarrow \infty} \lambda_{n}<\lim \sup _{n \rightarrow \infty} \lambda_{n}<2 \alpha$ and $\sum_{n=1}^{\infty}\left|\lambda_{n-1}-\lambda_{n}\right|<\infty$.

Then the sequence $\left\{x_{n}\right\}$ generated by Algorithm (42) converges strongly to $x^{*} \in \Omega^{*} \cap \Lambda \cap$ $F(T)$, which is the unique solution of the variational inequality

$$
\left\langle\frac{1}{\tau}(I-f) x^{*}+(I-S) x^{*}, x-x^{*}\right\rangle \geq 0, \quad \forall x \in \Omega^{*} \cap \Lambda \cap F(T) .
$$

Proof From $\lim _{n \rightarrow \infty}\left(\beta_{n} / \alpha_{n}\right)=\tau \in(0, \infty)$, without loss of generality, we can assume that $\beta_{n} \leq(1+\tau) \alpha_{n}$ for all $n \geq 1$. Hence $\beta_{n} \rightarrow 0$. By a similar argument as that in Lemmas 3.1 and 3.2, we can deduce that $\left\{x_{n}\right\}$ is bounded, $\lim _{n \rightarrow \infty}\left\|x_{n+1}-x_{n}\right\|=0, \lim _{n \rightarrow \infty}\left\|x_{n}-z_{n}\right\|=0$ $\left(\right.$ see (38)) and $\left(I-V_{i}\right) x_{n} \rightarrow 0$. Then we have

$$
\left\|y_{n}-x_{n}\right\| \leq \beta_{n}\left\|x_{n}-S x_{n}\right\|+\left(1-\beta_{n}\right)\left\|x_{n}-z_{n}\right\| \rightarrow 0 \quad \text { as } n \rightarrow \infty .
$$


It follows that for all $i \geq 1$,

$$
\left\|y_{n}-V_{i} x_{n}\right\| \leq\left\|y_{n}-x_{n}\right\|+\left\|x_{n}-V_{i} x_{n}\right\| \rightarrow 0 \quad \text { as } n \rightarrow \infty .
$$

From (44) and (45), we have

$$
\left\|y_{n}-V_{i} y_{n}\right\| \leq\left\|y_{n}-V_{i} x_{n}\right\|+\left\|V_{i} x_{n}-V_{i} y_{n}\right\| \leq\left\|y_{n}-V_{i} x_{n}\right\|+\left\|y_{n}-x_{n}\right\| \rightarrow 0 \quad \text { as } n \rightarrow \infty .
$$

Set $w_{n}=\alpha_{n} f\left(x_{n}\right)+\sum_{i=1}^{n}\left(\alpha_{i-1}-\alpha_{i}\right) V_{i} y_{n}$. From (32) and (33), we obtain

$$
\begin{aligned}
\frac{\left\|x_{n+1}-x_{n}\right\|}{\beta_{n}} \leq & \frac{\left\|w_{n}-w_{n-1}\right\|}{\beta_{n}} \\
\leq & \left(1-(1-\rho) \alpha_{n}\right) \frac{\left\|x_{n}-x_{n-1}\right\|}{\beta_{n}} \\
& +M\left(\frac{1}{\mu} \frac{\left|r_{n}-r_{n-1}\right|}{\beta_{n}}+\frac{\left|\lambda_{n}-\lambda_{n-1}\right|}{\beta_{n}}+\frac{\left|\beta_{n}-\beta_{n-1}\right|}{\beta_{n}}+\frac{\left|\alpha_{n}-\alpha_{n-1}\right|}{\beta_{n}}\right) \\
= & \left(1-(1-\rho) \alpha_{n}\right) \frac{|| x_{n}-x_{n-1} \|}{\beta_{n-1}}+\left(1-(1-\rho) \alpha_{n}\right)|| x_{n}-x_{n-1} \|\left(\frac{1}{\beta_{n}}-\frac{1}{\beta_{n-1}}\right) \\
& +M\left(\frac{1}{\mu} \frac{\left|r_{n}-r_{n-1}\right|}{\beta_{n}}+\frac{\left|\lambda_{n}-\lambda_{n-1}\right|}{\beta_{n}}+\frac{\left|\beta_{n}-\beta_{n-1}\right|}{\beta_{n}}+\frac{\left|\alpha_{n}-\alpha_{n-1}\right|}{\beta_{n}}\right) \\
\leq & \left(1-(1-\rho) \alpha_{n}\right) \frac{|| x_{n}-x_{n-1} \|}{\beta_{n-1}}+\left\|x_{n}-x_{n-1}\right\|\left|\frac{1}{\beta_{n}}-\frac{1}{\beta_{n-1}}\right| \\
& +M\left(\frac{1}{\mu} \frac{\left|r_{n}-r_{n-1}\right|}{\beta_{n}}+\frac{\left|\lambda_{n}-\lambda_{n-1}\right|}{\beta_{n}}+\frac{\left|\beta_{n}-\beta_{n-1}\right|}{\beta_{n}}+\frac{\left|\alpha_{n}-\alpha_{n-1}\right|}{\beta_{n}}\right) \\
\leq & \left(1-(1-\rho) \alpha_{n}\right) \frac{\mid x_{n}-x_{n-1} \|}{\beta_{n-1}}+\alpha_{n} K\left\|x_{n}-x_{n-1}\right\| \\
& +M\left(\frac{1}{\mu} \frac{\left|r_{n}-r_{n-1}\right|}{\beta_{n}}+\frac{\left|\lambda_{n}-\lambda_{n-1}\right|}{\beta_{n}}+\frac{\left|\beta_{n}-\beta_{n-1}\right|}{\beta_{n}}+\frac{\left|\alpha_{n}-\alpha_{n-1}\right|}{\beta_{n}}\right) \\
\leq & \left(1-(1-\rho) \alpha_{n}\right) \frac{\left\|w_{n-1}-w_{n-2}\right\|}{\beta_{n-1}}+\alpha_{n} K\left\|x_{n}-x_{n-1}\right\| \\
& +M\left(\frac{1}{\mu} \frac{\left|r_{n}-r_{n-1}\right|}{\beta_{n}}+\frac{\left|\lambda_{n}-\lambda_{n-1}\right|}{\beta_{n}}+\frac{\left|\beta_{n}-\beta_{n-1}\right|}{\beta_{n}}+\frac{\left|\alpha_{n}-\alpha_{n-1}\right|}{\beta_{n}}\right) .
\end{aligned}
$$

Let $\gamma_{n}=(1-\rho) \alpha_{n}$ and $\delta_{n}=\alpha_{n} K\left\|x_{n}-x_{n-1}\right\|+M\left(\frac{1}{\mu} \frac{\left|r_{n}-r_{n-1}\right|}{\beta_{n}}+\frac{\left|\lambda_{n}-\lambda_{n-1}\right|}{\beta_{n}}+\frac{\left|\beta_{n}-\beta_{n-1}\right|}{\beta_{n}}+\frac{\left|\alpha_{n}-\alpha_{n-1}\right|}{\beta_{n}}\right)$.

From conditions (a) and (d), we have

$$
\sum_{n=1}^{\infty} \gamma_{n}=\infty \text { and } \lim _{n \rightarrow \infty} \frac{\delta_{n}}{\gamma_{n}}=0
$$

By Lemma 2.6, we obtain

$$
\lim _{n \rightarrow \infty} \frac{\left\|x_{n+1}-x_{n}\right\|}{\beta_{n}}=0, \quad \lim _{n \rightarrow \infty} \frac{\left\|w_{n+1}-w_{n}\right\|}{\beta_{n}}=\lim _{n \rightarrow \infty} \frac{\left\|w_{n+1}-w_{n}\right\|}{\alpha_{n}}=0 .
$$


From (42), we have

$$
x_{n+1}=P_{C}\left[w_{n}\right]-w_{n}+\alpha_{n} f\left(x_{n}\right)+\sum_{i=1}^{n}\left(\alpha_{i-1}-\alpha_{i}\right)\left(V_{i} y_{n}-y_{n}\right)+\left(1-\alpha_{n}\right) y_{n}
$$

Hence it follows that

$$
\begin{aligned}
x_{n}-x_{n+1}= & \left(1-\alpha_{n}\right) x_{n}+\alpha_{n} x_{n} \\
& -\left(P_{C}\left[w_{n}\right]-w_{n}+\alpha_{n} f\left(x_{n}\right)+\sum_{i=1}^{n}\left(\alpha_{i-1}-\alpha_{i}\right)\left(V_{i} y_{n}-y_{n}\right)+\left(1-\alpha_{n}\right) y_{n}\right) \\
= & \left(1-\alpha_{n}\right)\left[\beta_{n}\left(x_{n}-S x_{n}\right)+\left(1-\beta_{n}\right)\left(x_{n}-z_{n}\right)\right]+\left(w_{n}-P_{C}\left[w_{n}\right]\right) \\
& +\sum_{i=1}^{n}\left(\alpha_{i-1}-\alpha_{i}\right)\left(y_{n}-V_{i} y_{n}\right)+\alpha_{n}\left(x_{n}-f\left(x_{n}\right)\right),
\end{aligned}
$$

and hence

$$
\begin{aligned}
\frac{x_{n}-x_{n+1}}{\left(1-\alpha_{n}\right) \beta_{n}}= & x_{n}-S x_{n}+\frac{\left(1-\beta_{n}\right)}{\beta_{n}}\left(x_{n}-z_{n}\right)+\frac{1}{\left(1-\alpha_{n}\right) \beta_{n}}\left(w_{n}-P_{C}\left[w_{n}\right]\right) \\
& +\frac{1}{\left(1-\alpha_{n}\right) \beta_{n}} \sum_{i=1}^{n}\left(\alpha_{i-1}-\alpha_{i}\right)\left(y_{n}-V_{i} y_{n}\right)+\frac{\alpha_{n}}{\left(1-\alpha_{n}\right) \beta_{n}}\left(x_{n}-f\left(x_{n}\right)\right) .
\end{aligned}
$$

Let $v_{n}=\frac{x_{n}-x_{n+1}}{\left(1-\alpha_{n}\right) \beta_{n}}$. For any $z \in \Omega^{*} \cap \Lambda \cap F(T)$, we have

$$
\begin{aligned}
\left\langle v_{n}, x_{n}-z\right\rangle= & \frac{1}{\left(1-\alpha_{n}\right) \beta_{n}}\left\langle w_{n}-P_{C}\left[w_{n}\right], P_{C}\left[w_{n-1}\right]-z\right\rangle+\frac{\alpha_{n}}{\left(1-\alpha_{n}\right) \beta_{n}}\left\langle(I-f) x_{n}, x_{n}-z\right\rangle \\
& +\left\langle x_{n}-S x_{n}, x_{n}-z\right\rangle+\frac{\left(1-\beta_{n}\right)}{\beta_{n}}\left\langle x_{n}-z_{n}, x_{n}-z\right\rangle \\
& +\frac{1}{\left(1-\alpha_{n}\right) \beta_{n}} \sum_{i=1}^{n}\left(\alpha_{i-1}-\alpha_{i}\right)\left\langle y_{n}-V_{i} y_{n}, x_{n}-z\right\rangle .
\end{aligned}
$$

Since $S$ is a nonexpansive mapping, $f$ is a $\rho$-contraction mapping and $V_{i}$ is a $k_{i}$-strict pseudo-contraction mapping. Then $(I-S)$ and $\left(I-V_{i}\right)$ are monotone and $f$ is strongly monotone with a coefficient $(1-\rho)$. We can deduce

$$
\begin{aligned}
\left\langle x_{n}-S x_{n}, x_{n}-z\right\rangle & =\left\langle(I-S) x_{n}-(I-S) z, x_{n}-z\right\rangle+\left\langle(I-S) z, x_{n}-z\right\rangle \\
& \geq\left\langle(I-S) z, x_{n}-z\right\rangle, \\
\left\langle(I-f) x_{n}, x_{n}-z\right\rangle & =\left\langle(I-f) x_{n}-(I-f) z, x_{n}-z\right\rangle+\left\langle(I-f) z, x_{n}-z\right\rangle \\
& \geq(1-\rho)\left\|x_{n}-z\right\|^{2}+\left\langle(I-f) z, x_{n}-z\right\rangle, \\
\left\langle\left(I-V_{i}\right) y_{n}, x_{n}-z\right\rangle & =\left\langle\left(I-V_{i}\right) y_{n}-\left(I-V_{i}\right) z, x_{n}-y_{n}\right\rangle+\left\langle\left(I-V_{i}\right) y_{n}-\left(I-V_{i}\right) z, y_{n}-z\right\rangle \\
& \geq\left\langle\left(I-V_{i}\right) y_{n}-\left(I-V_{i}\right) z, x_{n}-y_{n}\right\rangle \\
& =\left\langle\left(I-V_{i}\right) y_{n}, x_{n}-y_{n}\right\rangle \\
& =\left\langle\left(I-V_{i}\right) y_{n}, \beta_{n}\left(x_{n}-S x_{n}\right)+\left(1-\beta_{n}\right)\left(x_{n}-z_{n}\right)\right\rangle .
\end{aligned}
$$


From (16), we get

$$
\begin{aligned}
& \left\langle w_{n}-P_{C}\left[w_{n}\right], P_{C}\left[w_{n-1}\right]-z\right\rangle \\
& \quad=\left\langle w_{n}-P_{C}\left[w_{n}\right], P_{C}\left[w_{n-1}\right]-P_{C}\left[w_{n}\right]\right\rangle+\left\langle w_{n}-P_{C}\left[w_{n}\right], P_{C}\left[w_{n}\right]-z\right\rangle \\
& \quad \geq\left\langle w_{n}-P_{C}\left[w_{n}\right], P_{C}\left[w_{n-1}\right]-P_{C}\left[w_{n}\right]\right\rangle .
\end{aligned}
$$

Then, from (46)-(49), we have

$$
\begin{aligned}
\left\langle v_{n}, x_{n}-z\right\rangle \geq & \frac{1}{\left(1-\alpha_{n}\right) \beta_{n}}\left\langle w_{n}-P_{C}\left[w_{n}\right], P_{C}\left[w_{n-1}\right]-P_{C}\left[w_{n}\right]\right\rangle+\frac{\alpha_{n}}{\left(1-\alpha_{n}\right) \beta_{n}}\left\langle(I-f) z, x_{n}-z\right\rangle \\
& +\left\langle(I-S) z, x_{n}-z\right\rangle+\frac{\left(1-\beta_{n}\right)}{\beta_{n}}\left\langle x_{n}-z_{n}, x_{n}-z\right\rangle \\
& +\frac{\left(1-\beta_{n}\right)}{\left(1-\alpha_{n}\right) \beta_{n}} \sum_{i=1}^{n}\left(\alpha_{i-1}-\alpha_{i}\right)\left\langle\left(I-V_{i}\right) y_{n}, x_{n}-z_{n}\right\rangle \\
& +\frac{1}{\left(1-\alpha_{n}\right)} \sum_{i=1}^{n}\left(\alpha_{i-1}-\alpha_{i}\right)\left\langle\left(I-V_{i}\right) y_{n}, x_{n}-S x_{n}\right\rangle+\frac{(1-\rho) \alpha_{n}}{\left(1-\alpha_{n}\right) \beta_{n}}\left\|x_{n}-z\right\|^{2} .
\end{aligned}
$$

Then we obtain

$$
\begin{aligned}
& \left\|x_{n}-z\right\|^{2} \leq \frac{1}{(1-\rho) \alpha_{n}}\left\|w_{n}-P_{C}\left[w_{n}\right]\right\|\left\|w_{n-1}-w_{n}\right\|-\frac{1}{(1-\rho)}\left\langle(I-f) z, x_{n}-z\right\rangle \\
& +\frac{\left(1-\alpha_{n}\right) \beta_{n}}{(1-\rho) \alpha_{n}}\left(\left\langle v_{n}, x_{n}-z\right\rangle-\left\langle(I-S) z, x_{n}-z\right\rangle\right) \\
& -\frac{\left(1-\beta_{n}\right)\left(1-\alpha_{n}\right)}{(1-\rho) \alpha_{n}}\left\langle x_{n}-z_{n}, x_{n}-z\right\rangle \\
& -\frac{\left(1-\beta_{n}\right)}{(1-\rho) \alpha_{n}} \sum_{i=1}^{n}\left(\alpha_{i-1}-\alpha_{i}\right)\left\langle\left(I-V_{i}\right) y_{n}, x_{n}-z_{n}\right\rangle \\
& -\frac{\beta_{n}}{(1-\rho) \alpha_{n}} \sum_{i=1}^{n}\left(\alpha_{i-1}-\alpha_{i}\right)\left\langle\left(I-V_{i}\right) y_{n}, x_{n}-S x_{n}\right\rangle \\
& \leq \frac{\left\|w_{n-1}-w_{n}\right\|}{(1-\rho) \alpha_{n}}\left\|w_{n}-P_{C}\left[w_{n}\right]\right\|-\frac{1}{(1-\rho)}\left\langle(I-f) z, x_{n}-z\right\rangle \\
& +\frac{\left(1-\alpha_{n}\right) \beta_{n}}{(1-\rho) \alpha_{n}}\left(\left\langle v_{n}, x_{n}-z\right\rangle-\left\langle(I-S) z, x_{n}-z\right\rangle\right) \\
& +\frac{1}{(1-\rho)} \frac{\left(1-\beta_{n}\right)}{\beta_{n}} \frac{\beta_{n}}{\alpha_{n}}\left\|x_{n}-z_{n}\right\|\left\|x_{n}-z\right\| \\
& +\frac{1}{(1-\rho)} \frac{\left(1-\beta_{n}\right)}{\beta_{n}} \frac{\beta_{n}}{\alpha_{n}} \sum_{i=1}^{n}\left(\alpha_{i-1}-\alpha_{i}\right)\left\|\left(I-V_{i}\right) y_{n}\right\|\left\|x_{n}-z_{n}\right\| \\
& -\frac{\beta_{n}}{(1-\rho) \alpha_{n}} \sum_{i=1}^{n}\left(\alpha_{i-1}-\alpha_{i}\right)\left\langle\left(I-V_{i}\right) y_{n}, x_{n}-S x_{n}\right\rangle \text {. }
\end{aligned}
$$

By condition (e) of Theorem 3.2, there exists a constant $N>0$ such that $\frac{1-\beta_{n}}{\beta_{n}} \leq N$. Since $\lim _{n \rightarrow \infty}\left\|x_{n}-z_{n}\right\|=0, v_{n} \rightarrow 0,\left(I-V_{i}\right) y_{n} \rightarrow 0$ and $\frac{\left\|w_{n-1}-w_{n}\right\|}{\alpha_{n}} \rightarrow 0$ as $n \rightarrow \infty$, then every weak cluster point of $\left\{x_{n}\right\}$ is also a strong cluster point. Since $\left\{x_{n}\right\}$ is bounded, by 
Lemma 3.2 there exists a subsequence $\left\{x_{n_{k}}\right\}$ of $\left\{x_{n}\right\}$ converging to a point $x^{*} \in F(T)$, and by some similar arguments in Theorem 3.1, we can show that $x^{*} \in \Omega^{*} \cap \Lambda \cap F(T)$.

From (46)-(49), it follows that for any $z \in \Omega^{*} \cap \Lambda \cap F(T)$,

$$
\begin{aligned}
& \left\langle(I-f) x_{n_{k}}, x_{n_{k}}-z\right\rangle \\
& =\frac{\left(1-\alpha_{n_{k}}\right) \beta_{n_{k}}}{\alpha_{n_{k}}}\left\langle v_{n_{k}}, x_{n_{k}}-z\right\rangle-\frac{1}{\alpha_{n_{k}}}\left\langle w_{n_{k}}-P_{C}\left[w_{n_{k}}\right], P_{C}\left[w_{n_{k}-1}\right]-z\right\rangle \\
& \quad-\frac{\left(1-\alpha_{n_{k}}\right) \beta_{n_{k}}}{\alpha_{n_{k}}}\left\langle x_{n_{k}}-S x_{n_{k}}, x_{n_{k}}-z\right\rangle-\frac{\left(1-\alpha_{n_{k}}\right)\left(1-\beta_{n_{k}}\right)}{\alpha_{n_{k}}}\left\langle x_{n_{k}}-z_{n_{k}}, x_{n_{k}}-z\right\rangle \\
& \quad-\frac{1}{\alpha_{n_{k}}} \sum_{i=1}^{n}\left(\alpha_{i-1}-\alpha_{i}\right)\left\langle y_{n_{k}}-V_{i} y_{n_{k}}, x_{n_{k}}-z\right\rangle \\
& \leq \\
& \quad \frac{\left(1-\alpha_{n_{k}}\right) \beta_{n_{k}}}{\alpha_{n_{k}}}\left\langle v_{n_{k}}, x_{n_{k}}-z\right\rangle+\frac{1}{\alpha_{n_{k}}}\left\|w_{n_{k}}-P_{C}\left[w_{n_{k}}\right]\right\|\left\|w_{n_{k}-1}-w_{n_{k}}\right\| \\
& \quad-\frac{\left(1-\alpha_{n_{k}}\right) \beta_{n_{k}}}{\alpha_{n_{k}}}\left\langle x_{n_{k}}-S x_{n_{k}}, x_{n_{k}}-z\right\rangle+\frac{\left(1-\beta_{n_{k}}\right)}{\beta_{n_{k}}} \frac{\beta_{n_{k}}}{\alpha_{n_{k}}}\left\|x_{n_{k}}-z_{n_{k}}\right\|\left\|x_{n_{k}}-z\right\| \\
& \quad-\frac{\left(1-\beta_{n_{k}}\right)}{\beta_{n_{k}}} \frac{\beta_{n_{k}}}{\alpha_{n_{k}}} \sum_{i=1}^{n_{k}}\left(\alpha_{i-1}-\alpha_{i}\right)\left\|\left(I-V_{i}\right) y_{n_{k}}\right\|\left\|x_{n_{k}}-z_{n_{k}}\right\| \\
& \quad-\frac{\beta_{n_{k}}}{\alpha_{n_{k}}} \sum_{i=1}^{n_{k}}\left(\alpha_{i-1}-\alpha_{i}\right)\left\langle\left(I-V_{i}\right) y_{n_{k}}, x_{n_{k}}-S x_{n_{k}}\right\rangle .
\end{aligned}
$$

Since $\lim _{n \rightarrow \infty}\left\|x_{n}-z_{n}\right\|=0, v_{n} \rightarrow 0,\left(I-V_{i}\right) y_{n} \rightarrow 0$ and $\frac{\left\|w_{n-1}-w_{n}\right\|}{\alpha_{n}} \rightarrow 0$, letting $k \rightarrow \infty$ in (50), we obtain

$$
\left\langle(I-f) x^{*}, x^{*}-z\right\rangle \leq-\tau\left\langle x^{*}-S x^{*}, x^{*}-z\right\rangle
$$

i.e.,

$$
\left\langle\frac{1}{\tau}(I-f) x^{*}+(I-S) x^{*}, z-x^{*}\right\rangle \geq 0
$$

In the following, we show that (43) has a unique solution. Assume that $x^{\prime}$ is another solution. Then we have

$$
\begin{aligned}
& \left\langle(I-f) x^{\prime}, x^{\prime}-x^{*}\right\rangle \leq-\tau\left\langle x^{\prime}-S x^{\prime}, x^{\prime}-x^{*}\right\rangle, \\
& \left\langle(I-f) x^{*}, x^{*}-x^{\prime}\right\rangle \leq-\tau\left\langle x^{*}-S x^{*}, x^{*}-x^{\prime}\right\rangle .
\end{aligned}
$$

Adding (51) and (52), we get

$$
\begin{aligned}
(1-\rho)\left\|x^{\prime}-x^{*}\right\|^{2} & \leq\left\langle(I-f) x^{\prime}-(I-f) x^{*}, x^{\prime}-x^{*}\right\rangle \\
& \leq-\tau\left\langle(I-S) x^{\prime}-(I-S) x^{*}, x^{\prime}-x^{*}\right\rangle \\
& \leq 0 .
\end{aligned}
$$


Then $x^{\prime}=x^{*}$. Since (43) has a unique solution, it follows that $w_{w}\left(x_{n}\right)=\left\{x^{*}\right\}$. Since every weak cluster point of $\left\{x_{n}\right\}$ is also a strong cluster point, we conclude that $\left\{x_{n}\right\} \rightarrow x^{*}$. This completes the proof.

\section{Applications}

In this section, we obtain the following results by using a special case of the proposed method. The first result can be viewed as an extension and improvement of the method of Gu et al. [11] for finding an approximate element of the common set of solutions of a split equilibrium problem and a hierarchical fixed point problem in a real Hilbert space.

Corollary 4.1 Let $H_{1}$ and $H_{2}$ be two real Hilbert spaces and $C \subseteq H_{1}$ and $Q \subseteq H_{2}$ be nonempty closed convex subsets of Hilbert spaces $H_{1}$ and $H_{2}$, respectively. Let $A: H_{1} \rightarrow H_{2}$ be a bounded linear operator. Let $D: C \rightarrow H_{1}$ be an $\alpha$-inverse strongly monotone mapping. Assume that $F_{1}: C \times C \rightarrow \mathbb{R}$ and $F_{2}: Q \times Q \rightarrow \mathbb{R}$ are the bifunctions satisfying Assumption 2.1 and $F_{2}$ is upper semicontinuous in the first argument. Let $S: C \rightarrow H_{1}$ be a nonexpansive mapping and $\left\{T_{i}\right\}_{i=1}^{\infty}: C \rightarrow C$ be a countable family of $k_{i}$-strict pseudo-contraction mappings such that $F(T) \cap \Omega^{*} \cap \Lambda \neq \emptyset$, where $F(T)=\bigcap_{i=1}^{\infty} F\left(T_{i}\right)$. Let $f$ be a $\rho$-contraction mapping. For a given $x_{0} \in C$ arbitrarily, let the iterative sequences $\left\{u_{n}\right\},\left\{x_{n}\right\},\left\{y_{n}\right\}$ and $\left\{z_{n}\right\}$ be generated by

$$
\begin{aligned}
& u_{n}=T_{r_{n}}^{F_{1}}\left(x_{n}+\gamma A^{*}\left(T_{r_{n}}^{F_{2}}-I\right) A x_{n}\right) ; \\
& y_{n}=\beta_{n} S x_{n}+\left(1-\beta_{n}\right) u_{n} ; \\
& x_{n+1}=P_{C}\left[\alpha_{n} f\left(x_{n}\right)+\sum_{i=1}^{n}\left(\alpha_{i-1}-\alpha_{i}\right) T_{i} y_{n}\right], \quad \forall n \geq 0,
\end{aligned}
$$

where $\left\{r_{n}\right\} \subset(0, \infty)$ and $\gamma \in(0,1 / L), L$ is the spectral radius of the operator $A^{*} A$ and $A^{*}$ is the adjoint of $A$ and $\alpha_{0}=1,\left\{\alpha_{n}\right\}$ is a strictly decreasing sequence in $(0,1)$ and $\left\{\beta_{n}\right\}$ is a sequence in $(0,1)$ satisfying the following conditions:

(a) $\lim _{n \rightarrow \infty} \alpha_{n}=0$ and $\sum_{n=1}^{\infty} \alpha_{n}=\infty$,

(b) $\lim _{n \rightarrow \infty} \frac{\beta_{n}}{\alpha_{n}}=\tau \in(0, \infty)$,

(c) $\sum_{n=1}^{\infty}\left(\alpha_{n-1}-\alpha_{n}\right)<\infty$ and $\sum_{n=1}^{\infty}\left|\beta_{n-1}-\beta_{n}\right|<\infty$,

(d) $\lim _{n \rightarrow \infty} \frac{\frac{1}{\mu}\left|r_{n}-r_{n-1}\right|+\left|\alpha_{n-1}-\alpha_{n}\right|+\left|\beta_{n-1}-\beta_{n}\right|}{\alpha_{n} \beta_{n}}=0$,

(e) there exists a constant $K>0$ such that $\frac{1}{\alpha_{n}}\left|\frac{1}{\beta_{n}}-\frac{1}{\beta_{n-1}}\right| \leq K$,

(f) $\liminf _{n \rightarrow \infty} r_{n}>0$ and $\sum_{n=1}^{\infty}\left|r_{n-1}-r_{n}\right|<\infty$.

Then the sequence $\left\{x_{n}\right\}$ generated by Algorithm (53) converges strongly to $x^{*} \in \Lambda \cap F(T)$, which is the unique solution of the variational inequality

$$
\left\langle\frac{1}{\tau}(I-f) x^{*}+(I-S) x^{*}, x-x^{*}\right\rangle \geq 0, \quad \forall x \in \Lambda \cap F(T) .
$$

Proof Put $\lambda_{n}=0$ and $k_{i}=0, \forall i \geq 1$ in Theorem 3.2. Then conclusion of Corollary 4.1 is obtained.

The following result can be viewed as an extension and improvement of the method of Yao et al. [27] for finding an approximate element of the common set of solutions of a split equilibrium problem and a hierarchical fixed point problem in a real Hilbert space. 
Corollary 4.2 Let $H_{1}$ and $H_{2}$ be two real Hilbert spaces and $C \subseteq H_{1}$ and $Q \subseteq H_{2}$ be nonempty closed convex subsets of Hilbert spaces $H_{1}$ and $H_{2}$, respectively. Let $A: H_{1} \rightarrow H_{2}$ be a bounded linear operator. Let $D: C \rightarrow H_{1}$ be an $\alpha$-inverse strongly monotone mapping. Assume that $F_{1}: C \times C \rightarrow \mathbb{R}$ and $F_{2}: Q \times Q \rightarrow \mathbb{R}$ are the bifunctions satisfying Assumption 2.1 and $F_{2}$ is upper semicontinuous in the first argument. Let $S: C \rightarrow H_{1}$ be a nonexpansive mapping and $T: C \rightarrow C$ be a $k$-strict pseudo-contraction mapping such that $F(T) \cap \Lambda \neq \emptyset$. Let $f$ be a $\rho$-contraction mapping. For a given $x_{0} \in C$ arbitrarily, let the iterative sequences $\left\{u_{n}\right\},\left\{x_{n}\right\},\left\{y_{n}\right\}$ and $\left\{z_{n}\right\}$ be generated by

$$
\begin{aligned}
& u_{n}=T_{r_{n}}^{F_{1}}\left(x_{n}+\gamma A^{*}\left(T_{r_{n}}^{F_{2}}-I\right) A x_{n}\right) ; \\
& y_{n}=\beta_{n} S x_{n}+\left(1-\beta_{n}\right) u_{n} ; \\
& x_{n+1}=P_{C}\left[\alpha_{n} f\left(x_{n}\right)+\left(1-\alpha_{n}\right) T y_{n}\right], \quad \forall n \geq 0,
\end{aligned}
$$

where $\left\{r_{n}\right\} \subset(0, \infty)$ and $\gamma \in(0,1 / L), L$ is the spectral radius of the operator $A^{*} A$ and $A^{*}$ is the adjoint of $A$ and $\alpha_{0}=1,\left\{\alpha_{n}\right\}$ is a strictly decreasing sequence in $(0,1)$ and $\left\{\beta_{n}\right\}$ is a sequence in $(0,1)$ satisfying the following conditions:

(a) $\lim _{n \rightarrow \infty} \alpha_{n}=0$ and $\sum_{n=1}^{\infty} \alpha_{n}=\infty$

(b) $\lim _{n \rightarrow \infty} \frac{\beta_{n}}{\alpha_{n}}=\tau \in(0, \infty)$,

(c) $\sum_{n=1}^{\infty}\left(\alpha_{n-1}-\alpha_{n}\right)<\infty$ and $\sum_{n=1}^{\infty}\left|\beta_{n-1}-\beta_{n}\right|<\infty$,

(d) $\lim _{n \rightarrow \infty} \frac{\frac{1}{\mu}\left|r_{n}-r_{n-1}\right|+\left|\alpha_{n-1}-\alpha_{n}\right|+\left|\beta_{n-1}-\beta_{n}\right|}{\alpha_{n} \beta_{n}}=0$,

(e) there exists a constant $K>0$ such that $\frac{1}{\alpha_{n}}\left|\frac{1}{\beta_{n}}-\frac{1}{\beta_{n-1}}\right| \leq K$,

(f) $\liminf _{n \rightarrow \infty} r_{n}>0$ and $\sum_{n=1}^{\infty}\left|r_{n-1}-r_{n}\right|<\infty$.

Then the sequence $\left\{x_{n}\right\}$ generated by Algorithm (54) converges strongly to $x^{*} \in \Lambda \cap F(T)$, which is the unique solution of the variational inequality

$$
\left\langle\frac{1}{\tau}(I-f) x^{*}+(I-S) x^{*}, x-x^{*}\right\rangle \geq 0, \quad \forall x \in \Lambda \cap F(T) .
$$

Proof Put $\lambda_{n}=0, k_{i}=0$ and $T_{i}=T, \forall i \geq 1$ in Theorem 3.2. Then conclusion of Corollary 4.2 is obtained.

\section{Competing interests}

The author declares that they have no competing interests.

Received: 21 June 2013 Accepted: 1 October 2013 Published: 08 Nov 2013

\section{References}

1. Acedo, GL, Xu, HK: Iterative methods for strictly pseudo-contractions in Hilbert space. Nonlinear Anal. 67, 2258-2271 (2007)

2. Blum, E, Oettli, W: From optimization and variational inequalities to equilibrium problems. Math. Stud. 63, 123-145 (1994)

3. Byrne, C, Censor, Y, Gibali, A, Reich, S: Weak and strong convergence of algorithms for the split common null point problem. arXiv:1108.5953

4. Censor, Y, Gibali, A, Reich, S: Algorithms for the split variational inequality problem. Numer. Algorithms 59(2), 301-323 (2012)

5. Cianciaruso, F, Marino, G, Muglia, L, Yao, Y: On a two-steps algorithm for hierarchical fixed point problems and variational inequalities. J. Inequal. Appl. 2009, Article ID 208692 (2009)

6. Cianciaruso, F, Marino, G, Muglia, L, Yao, Y: A hybrid projection algorithm for finding solutions of mixed equilibrium problem and variational inequality problem. Fixed Point Theory Appl. 2010, Article ID 383740 (2010)

7. Chang, SS, Lee, HWJ, Chan, CK: A new method for solving equilibrium problem fixed point problem and variational inequality problem with application to optimization. Nonlinear Anal. 70, 3307-3319 (2009) 
8. Combettes, PL, Hirstoaga, SA: Equilibrium programming using proximal like algorithms. Math. Program. 78, 29-41 (1997)

9. Crombez, G: A geometrical look at iterative methods for operators with fixed points. Numer. Funct. Anal. Optim. 26, 157-175 (2005)

10. Crombez, G: A hierarchical presentation of operators with fixed points on Hilbert spaces. Numer. Funct. Anal. Optim. 27, 259-277 (2006)

11. Gu, G, Wang, S, Cho, YJ: Strong convergence algorithms for hierarchical fixed points problems and variational inequalities. J. Appl. Math. 2011, Article ID 164978 (2011)

12. Katchang, P, Kumam, P: A new iterative algorithm for equilibrium problems, variational inequalities and fixed point problems in a Hilbert space. Appl. Math. Comput. 32, 19-38 (2010)

13. Kazmi, KR, Rizvi, SH: Iterative approximation of a common solution of a split equilibrium problem, a variational inequality problem and a fixed point problem. J. Egypt. Math. Soc. 21, 44-51 (2013)

14. Lions, JL, Stampacchia, G: Variational inequalities. Commun. Pure Appl. Math. 20, 493-512 (1967)

15. Mainge, PE, Moudafi, A: Strong convergence of an iterative method for hierarchical fixed-point problems. Pac. J. Optim. 3(3), 529-538 (2007)

16. Marino, G, Xu, HK: Convergence of generalized proximal point algorithms. Commun. Pure Appl. Anal. 3, 791-808 (2004)

17. Marino, G, Xu, HK: A general iterative method for nonexpansive mappings in Hilbert spaces. J. Math. Anal. Appl. 318(1), 43-52 (2006)

18. Marino, G, Xu, HK: Explicit hierarchical fixed point approach to variational inequalities. J. Optim. Theory Appl. 149(1), 61-78 (2011)

19. Moudafi, A, Théra, M: Proximal and Dynamical Approaches to Equilibrium Problems. Lecture Notes in Economics and Mathematical Systems, vol. 477. Springer, New York (1999)

20. Moudafi, A: Mixed equilibrium problems sensitivity analysis and algorithmic aspect. Comput. Math. Appl. 44 1099-1108 (2002)

21. Moudafi, A: Krasnoselski-Mann iteration for hierarchical fixed-point problems. Inverse Probl. 23(4), $1635-1640$ (2007)

22. Moudafi, A: Split monotone variational inclusions. J. Optim. Theory Appl. 150, 275-283 (2011)

23. Plubtieng, S, Punpaeng, R: A general iterative method for equilibrium problems and fixed point problems in Hilbert spaces. J. Math. Anal. Appl. 336, 455-469 (2007)

24. Qin, X, Shang, M, Su, Y: A general iterative method for equilibrium problem and fixed point problem in Hilbert spaces Nonlinear Anal. 69, 3897-3909 (2008)

25. Rockafellar, RT: On the maximality of sums nonlinear monotone operators. Trans. Am. Math. Soc. 149, 75-88 (1970)

26. Xu, HK: Iterative algorithms for nonlinear operators. J. Lond. Math. Soc. 66, 240-256 (2002)

27. Yao, Y, Cho, YJ, Liou, YC: Iterative algorithms for hierarchical fixed points problems and variational inequalities. Math. Comput. Model. 52(9-10), 1697-1705 (2010)

28. Yao, Y, Liou, YC, Kang, SM: Approach to common elements of variational inequality problems and fixed point problems via a relaxed extragradient method. Comput. Math. Appl. 59(11), 3472-3480 (2010)

29. Zhou, $\mathrm{H}$ : Convergence theorems of fixed points for $\mathrm{k}$-strict pseudo-contractions in Hilbert spaces. Nonlinear Anal. 69 456-462 (2008)

10.1186/1687-1812-2013-278

Cite this article as: Bnouhachem: Algorithms of common solutions for a variational inequality, a split equilibrium problem and a hierarchical fixed point problem. Fixed Point Theory and Applications 2013, 2013:278

\section{Submit your manuscript to a SpringerOpen ${ }^{\ominus}$ journal and benefit from:}

- Convenient online submission

- Rigorous peer review

- Immediate publication on acceptance

- Open access: articles freely available online

- High visibility within the field

- Retaining the copyright to your article 